

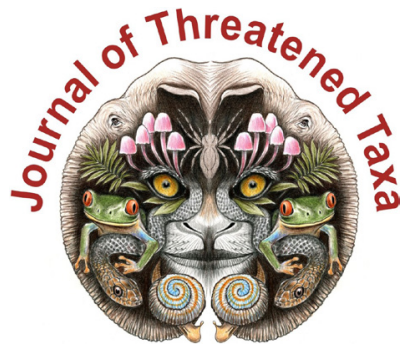

ISSN 0974-7907 (Online); ISSN $0974-7893$ (Print)

Publisher

Host

Wildlife Information Liaison Development Society

www.wild.zooreach.org

Zoo Outreach Organization www.zooreach.org

No. 12, Thiruvannamalai Nagar, Saravanampatti - Kalapatti Road, Saravanampatti, Coimbatore, Tamil Nadu 641035, India

Ph: +91 9385339863 | www.threatenedtaxa.org

Email: sanjay@threatenedtaxa.org

EDITORS

\section{Founder \& Chief Editor}

Dr. Sanjay Molur

Wildlife Information Liaison Development (WILD) Society \& Zoo Outreach Organization (ZOO),

12 Thiruvannamalai Nagar, Saravanampatti, Coimbatore, Tamil Nadu 641035, India

\section{Deputy Chief Editor}

Dr. Neelesh Dahanukar

Noida, Uttar Pradesh, India

\section{Managing Editor}

Mr. B. Ravichandran, WILD/ZOO, Coimbatore, India

\section{Associate Editors}

Dr. Mandar Paingankar, Government Science College Gadchiroli, Maharashtra 442605, India

Dr. Ulrike Streicher, Wildlife Veterinarian, Eugene, Oregon, USA

Ms. Priyanka Iyer, ZOO/WILD, Coimbatore, Tamil Nadu 641035, India

Dr. B.A. Daniel, ZOO/WILD, Coimbatore, Tamil Nadu 641035, India

\section{Editorial Board}

Dr. Russel Mittermeier

Executive Vice Chair, Conservation International, Arlington, Virginia 22202, USA

\section{Prof. Mewa Singh Ph.D., FASc, FNA, FNASc, FNAPsy}

Ramanna Fellow and Life-Long Distinguished Professor, Biopsychology Laboratory, and Institute of Excellence, University of Mysore, Mysuru, Karnataka 570006, India; Honorary Professor, Jawaharlal Nehru Centre for Advanced Scientific Research, Bangalore; and Adjunct Professor, National Institute of Advanced Studies, Bangalore

\section{Stephen D. Nash}

Scientific Illustrator, Conservation International, Dept. of Anatomical Sciences, Health Sciences Center, T-8, Room 045, Stony Brook University, Stony Brook, NY 11794-8081, USA

\section{Dr. Fred Pluthero}

Toronto, Canada

\section{Dr. Priya Davidar}

Sigur Nature Trust, Chadapatti, Mavinhalla PO, Nilgiris, Tamil Nadu 643223, India

\section{Dr. Martin Fisher}

Senior Associate Professor, Battcock Centre for Experimental Astrophysics, Cavendish

Laboratory, JJ Thomson Avenue, Cambridge CB3 OHE, UK

\section{Dr. John Fellowes}

Honorary Assistant Professor, The Kadoorie Institute, 8/F, T.T. Tsui Building, The University of Hong Kong, Pokfulam Road, Hong Kong

\section{Prof. Dr. Mirco Solé}

Universidade Estadual de Santa Cruz, Departamento de Ciências Biológicas, Vice-coordenado do Programa de Pós-Graduação em Zoologia, Rodovia Ilhéus/Itabuna, Km 16 (45662-000)

Salobrinho, Ilhéus - Bahia - Brasil

\section{Dr. Rajeev Raghavan}

Professor of Taxonomy, Kerala University of Fisheries \& Ocean Studies, Kochi, Kerala, India

\section{English Editors}

Mrs. Mira Bhojwani, Pune, India

Dr. Fred Pluthero, Toronto, Canad

Mr. P. Ilangovan, Chennai, India

Web Development

Mrs. Latha G. Ravikumar, ZOO/WILD, Coimbatore, India

\section{Typesetting}

Mr. Arul Jagadish, ZOO, Coimbatore, India

Mrs. Radhika, ZOO, Coimbatore, India

Mrs. Geetha, ZOO, Coimbatore India
Fundraising/Communications

Mrs. Payal B. Molur, Coimbatore, India

Subject Editors 2018-2020

Fungi

Dr. B. Shivaraju, Bengaluru, Karnataka, India

Dr. R.K. Verma, Tropical Forest Research Institute, Jabalpur, India

Dr. Vatsavaya S. Raju, Kakatiay University, Warangal, Andhra Pradesh, India

Dr. M. Krishnappa, Jnana Sahyadri, Kuvempu University, Shimoga, Karnataka, India

Dr. K.R. Sridhar, Mangalore University, Mangalagangotri, Mangalore, Karnataka, India

Dr. Gunjan Biswas, Vidyasagar University, Midnapore, West Bengal, India

\section{Plants}

Dr. G.P. Sinha, Botanical Survey of India, Allahabad, India

Dr. N.P. Balakrishnan, Ret. Joint Director, BSI, Coimbatore, India

Dr. Shonil Bhagwat, Open University and University of Oxford, UK

Prof. D.J. Bhat, Retd. Professor, Goa University, Goa, India

Dr. Ferdinando Boero, Università del Salento, Lecce, Italy

Dr. Dale R. Calder, Royal Ontaro Museum, Toronto, Ontario, Canada

Dr. Cleofas Cervancia, Univ. of Philippines Los Baños College Laguna, Philippines

Dr. F.B. Vincent Florens, University of Mauritius, Mauritius

Dr. Merlin Franco, Curtin University, Malaysia

Dr. V. Irudayaraj, St. Xavier's College, Palayamkottai, Tamil Nadu, India

Dr. B.S. Kholia, Botanical Survey of India, Gangtok, Sikkim, India

Dr. Pankaj Kumar, Kadoorie Farm and Botanic Garden Corporation, Hong Kong S.A.R., China

Dr. V. Sampath Kumar, Botanical Survey of India, Howrah, West Bengal, India

Dr. A.J. Solomon Raju, Andhra University, Visakhapatnam, India

Dr. Vijayasankar Raman, University of Mississippi, USA

Dr. B. Ravi Prasad Rao, Sri Krishnadevaraya University, Anantpur, India

Dr. K. Ravikumar, FRLHT, Bengaluru, Karnataka, India

Dr. Aparna Watve, Pune, Maharashtra, India

Dr. Qiang Liu, Xishuangbanna Tropical Botanical Garden, Yunnan, China

Dr. Noor Azhar Mohamed Shazili, Universiti Malaysia Terengganu, Kuala Terengganu, Malaysia

Dr. M.K. Vasudeva Rao, Shiv Ranjani Housing Society, Pune, Maharashtra, India

Prof. A.J. Solomon Raju, Andhra University, Visakhapatnam, India

Dr. Mandar Datar, Agharkar Research Institute, Pune, Maharashtra, India

Dr. M.K. Janarthanam, Goa University, Goa, India

Dr. K. Karthigeyan, Botanical Survey of India, India

Dr. Errol Vela, University of Montpellier, Montpellier, France

Dr. P. Lakshminarasimhan, Botanical Survey of India, Howrah, India

Dr. Larry R. Noblick, Montgomery Botanical Center, Miami, USA

Dr. K. Haridasan, Pallavur, Palakkad District, Kerala, India

Dr. Analinda Manila-Fajard, University of the Philippines Los Banos, Laguna, Philippines

Dr. P.A. Sinu, Central University of Kerala, Kasaragod, Kerala, India

Dr. Afroz Alam, Banasthali Vidyapith (accredited A grade by NAAC), Rajasthan, India

Dr. K.P. Rajesh, Zamorin's Guruvayurappan College, GA College PO, Kozhikode, Kerala, India

Dr. David E. Boufford, Harvard University Herbaria, Cambridge, MA 02138-2020, USA

Dr. Ritesh Kumar Choudhary, Agharkar Research Institute, Pune, Maharashtra, India

Dr. Navendu Page, Wildlife Institute of India, Chandrabani, Dehradun, Uttarakhand, India

\section{Invertebrates}

Dr. R.K. Avasthi, Rohtak University, Haryana, India

Dr. D.B. Bastawade, Maharashtra, India

Dr. Partha Pratim Bhattacharjee, Tripura University, Suryamaninagar, India

Dr. Kailash Chandra, Zoological Survey of India, Jabalpur, Madhya Pradesh, India

Dr. Ansie Dippenaar-Schoeman, University of Pretoria, Queenswood, South Africa

Dr. Rory Dow, National Museum of natural History Naturalis, The Netherlands

Dr. Brian Fisher, California Academy of Sciences, USA

Dr. Richard Gallon, llandudno, North Wales, LL30 1UP

Dr. Hemant V. Ghate, Modern College, Pune, India

Dr. M. Monwar Hossain, Jahangirnagar University, Dhaka, Bangladesh

Mr. Jatishwor Singh Irungbam, Biology Centre CAS, Branišovská, Czech Republic.

Dr. Ian J. Kitching, Natural History Museum, Cromwell Road, UK

Dr. George Mathew, Kerala Forest Research Institute, Peechi, India

For Focus, Scope, Aims, and Policies, visit https://threatenedtaxa.org/index.php/JoTT/aims_scope
For Article Submission Guidelines, visit https://threatenedtaxa.org/index.php/JoTT/about/submissions
For Policies against Scientific Misconduct, visit https://threatenedtaxa.org/index.php/JoTT/policies_various

continued on the back inside cover 


\title{
An enumeration of the flowering plants of Kyongnosla Alpine Sanctuary in eastern Sikkim, India
}

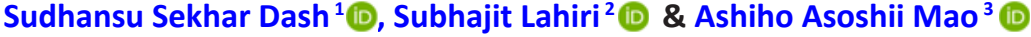 \\ ${ }^{1,3}$ Botanical Survey of India, III MSO Building, 5-6 th $^{\text {th }}$ loor, CGO Complex, DF Block, Sector-1, Salt Lake, West Bengal 700064, India. \\ ${ }^{2}$ Botanical Survey of India, Central National Herbarium, Howrah, Kolkata, West Bengal 711103, India. \\ ${ }^{1}$ ssdash2002@gmail.com (corresponding author), ${ }^{2}$ subhajitbsi@yahoo.com, ${ }^{3}$ aamao2008@gmail.com
}

\begin{abstract}
The present paper is the outcome of an extensive floristic survey conducted in two phases by the authors in Kyongnosla Alpine Sanctuary, East Sikkim, India. During the study 411 taxa (400 species, 04 subspecies and 07 varieties) belonging to 173 genera and 54 families were recorded. The most dominating family was Asteraceae with 44 species followed by Ericaceae 28 species, Ranunculaceae 26 species, Polygonaceae 24 species and Rosaceae 20 species. These five families represent $34.13 \%$ of the total taxa recorded from the sanctuary. About 12 families were represented by only one species each. The most dominating genus was Rhododendron (18 species) followed by Primula (16 species), Pedicularis (15 species), Gentiana (11 species), and Impatiens (10 species). Among the different growth forms, herbs contributed the maximum ( $86.61 \%)$ followed by shrubs $(7.79 \%)$, trees (4.87\%), climbers $0.49 \%$, and epiphyte $(0.24 \%)$.
\end{abstract}

Keywords: checklist, eastern Himalaya, floristic survey, growth form, Himalayan forest types.

Comprehensive documentation and identification of plant diversity is one of the targets of Global Strategy for Plant Conservation (GSPC). Being a signatory of the Convention on Plant Diversity (CBD), India is committed towards achieving a complete inventory of plant diversity of all the protected and nonprotected areas (Singh \& Dash 2015). India is endowed with diverse ecosystems ranging from high altitude cold deserts to hot and humid coastlands which show rich floral diversity. Continuous survey and exploration in different habitats have facilitated an updated inventory of plants, which opens up different potential linkages among various sectors and implementation of appropriate action on management and sustainable conservation.

Kyongnosla Alpine Sanctuary (KAS) situated in the East district of Sikkim (Figure 1) between $27^{\circ} 22^{\prime} 33^{\prime \prime} \mathrm{N}$ latitude and $88^{\circ} 44^{\prime} 13^{\prime \prime} E$ longitude covers an area of 31 $\mathrm{km}^{2}$ between the elevation ranging from $3,000-4,500 \mathrm{~m}$. In the initial notification 45/WL/83/625 dated 29.viii.84, the area demarcated was $4.5 \mathrm{~km}^{2}$, however in the second and final notification 45/WL/F/92/1585/F\&WL dated 12.v.92 the area of the sanctuary extended to $31 \mathrm{~km}^{2}$. The vegetation of the KAS comprises different ecological zones depending upon the elevation, viz., mixed Rhododendron temperate forest, coniferous forest along open slopes and ridges and alpine scrubs on upper reaches. Though it encompasses a small area, the sanctuary supports a luxuriant temperate and alpine vegetation owing to its unique topography, variation of altitudes and high annual precipitation. All representative Himalayan forest types (Champion

Citation: Dash, S.S., S. Lahiri \& A.A. Mao (2021). An enumeration of the flowering plants of Kyongnosla Alpine Sanctuary in eastern Sikkim, India. Journal of Threatened Taxa 13(13): 20098-20117. https://doi.org/10.11609/jott.7040.13.13.20098-20117

Copyright: @ Dash et al. 2021. Creative Commons Attribution 4.0 International License. JoTT allows unrestricted use, reproduction, and distribution of this article in any medium by providing adequate credit to the author(s) and the source of publication.

Funding: National Mission on Himalayan Studies (NMHS/2015-16/LG02/02).

Competing interests: The authors declare no competing interests.
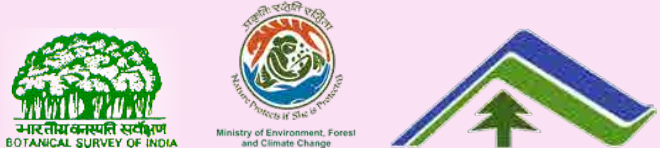

Acknowledgements: The authors are thankful to the director, Botanical Survey of India; scientist-in-charge, CAL, ASSAM and BSHC for providing necessary facilities and granting permission for herbarium consultation. The authors are also grateful to the secretary \& principal chief conservator of forests, Department of Forests, Govt. of Sikkim for providing survey permission. The financial support provided by the Ministry of Environment, Forest \& Climate Change (MoEF\&CC), New Delhi under 'National Mission on Himalayan Studies' (NMHS/2015-16/LG02/02) scheme is highly acknowledged. 
\& Seth 1968; Dash \& Singh 2010), such as northern montane wet temperate forests (dominated by Oaks Quercus, and Acer); Himalayan moist temperate forests (dominated by Rhododendron spp., Tsuga dumosa, Picea spinulosa, and Abies densa); Himalayan dry temperate forests (represented by gregarious growth of Juniperus wallichiana); sub-alpine forests (represented by Rhododendron spp., Betula utilis, Sorbus spp.) and alpine forests (gregarious patches of Rhododendron thickets, Juniperus recurva, and dense herbaceous growth) exist. The subalpine and alpine forests are present with climax formations, self-generating, resilience forest types. Nevertheless, this diversity is variously challenged by several driving forces such as over exploitation of medicinal plants, heavy grazing by yaks and developmental activities in the fringe areas. Extensive surveys of the study area were conducted to document the floristic composition in different altitudinal gradients of the KAS from July 2017-March 2020, being a part of our project work 'Biodiversity Assessment through Long-term Monitoring of Plots In the Indian Himalayan Landscape' under the National Mission of Himalayan Studies. In the paper we have enumerated 411 flowering plants occurring in the sanctuary with their correct accepted names.

\section{MATERIALS AND METHOdS}

The entire area of KAS was surveyed extensively in two phases. The first phase during 1998-2000 by the first author (SSD) and the second phase during 20172020 by the first two authors (SSD \& SL). More than 1,300 plant specimens were collected and processed following standard protocols (Jain \& Rao 1977). All the collected specimens were identified consulting available literature (Hooker 1872-1897; Grierson \& Long 1983; Long 1984; Grierson 1984; Grierson 1987; Grierson \& Long 1987; Hajra \& Verma 1996; Srivastava 1998; Aitken 1999; Aitken, Grierson \& Long 1999; Grierson \& Springate 2001; Lucksom 2007; Gogoi et al. 2018; Maity et al. 2018; Mao et al. 2018; Lahiri et al. 2019; Dash et al. 2020; Lahiri \& Dash 2020) and voucher specimens were deposited at BSHC and CAL for future reference. Earlier collections made from KAS by different collectors and available in different herbaria (ASSAM, BSHC, and CAL) were also taken into consideration while preparing the present enumeration. In the present inventory, families are arranged as per APG IV system of classification (Chase et al. 2016) and within the family, genera, and species are arranged alphabetically. For determination of current names, the World Flora Online (http:// www.worldfloraonline.org) and Plants of the World

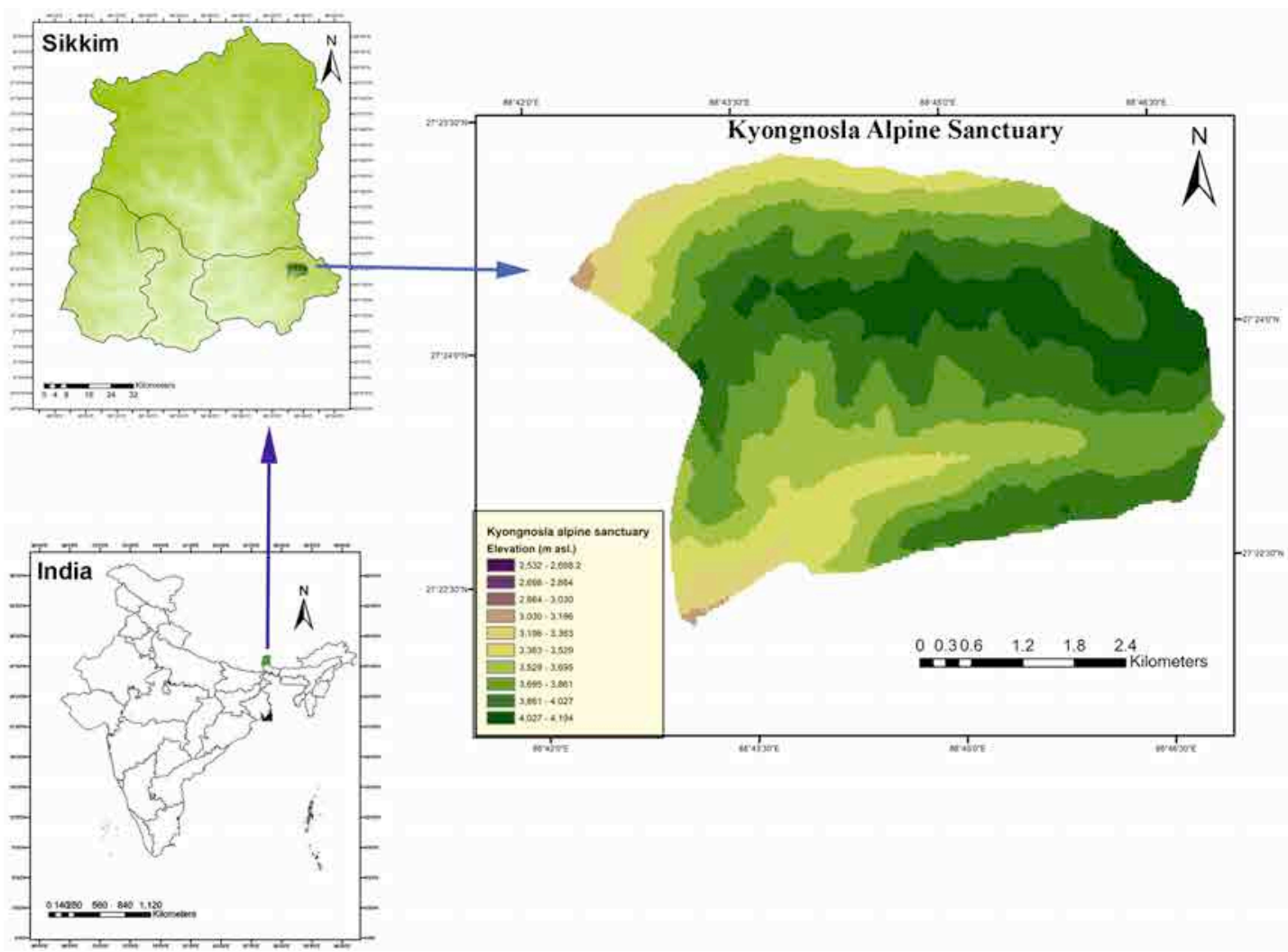

Figure 1. Location of Kyongnosla Alpine Sanctuary in eastern Sikkim. 
Online (http://www.plantsoftheworldonline.org) were consulted. The enumeration comprises family names, correct scientific names followed by valid author citation, growth form, altitude ranges where they occur and collection details along with the field number and herbarium acronym in each of the columns. Range of distribution of a species has been determined taking into consideration the present collections and also earlier collections deposited in different herbaria.

\section{RESULTS AND DISCUSSION}

The enumeration of the flowering plants of KAS includes 411 species belonging to 173 genera and 54 families (Table 2). The family Asteraceae with 44 species is the most dominant family, followed by Ericaceae (28 species), Ranunculaceae (26 species), Polygonaceae (24 species), and Rosaceae (20 species). These families represent $34.13 \%$ of the total species recorded from the sanctuary. Among the genera, Rhododendron is the most dominant genus represented by 18 species, followed by Primula (16 species), Gentiana (11 species), Impatiens (10 species), Corydalis (9 species), Saxifraga and Rhodiola (7 species each). Three families, i.e., Acanthaceae, Carlemanniaceae, and Euphorbiaceae are represented by only one species.

The plants present in the sanctuary show wider distribution in other parts of the Himalaya too, such as Allium prattii, Cyananthus lobatus, Gaultheria trichophylla, Juniperus recurva, Nardostachys jatamansii, Rosa sericea, and Streptopus simplex, which have a wider distribution from North-West Himalayas to China. The taxa showing eastward extension are Cassiope selaginoides, Gentiana stylophora, Morina nepalensis, Panax pseudo-ginseng, Primula sikkimensis, and Ribes luridum. Many species which are restricted only to eastern Himalaya are represented by Abies densa, Meconopsis villosa, Rhododendron lanatum, Impatiens cymbifera, Codonopsis benthamii, Codonopsis subsimplex, and Primula kingii.
The basal angiosperm is represented by only one species Schisandra grandiflora (Wall.) Hook.f. \& Thomson, while eudicots are represented by $85.88 \%$ and monocots by $13.86 \%$ of total species in KAS (Table 1 ).

The sanctuary provides a suitable habitat both for temperate and alpine species and holds many endangered and endemic species, viz. Aconitum ferox, Bhutanthera albomarginata, Ponerorchis puberula, Aconitum novoluridum, Codonopsis benthamii, Codonopsis subsimplex etc. The present study also recorded some of little-known species viz. Bhutanthera albomarginata, Ponerorchis puberula, Aconitum novoluridum, Codonopsis benthamii, Rubus lasiostylus, Sinopodophyllum hexandrum, Gentiana leucomelaena, Veratrilla burkilliana, Tibetoseris depressa, and Pedicularis porrecta. The sanctuary has been facing a continuous challenge in its community structure due to various anthropogenic interferences and heavy grazing which need to be controlled.

\section{REFERENCES}

Aitken, E. (1999). Family Gentianaceae, pp. 602-656. In: Long, D.G. (eds.). Flora of Bhutan Including a Record of Plants from Sikkim and Darjeeling Volume 2 (Part 2). Royal Botanic Garden Edinburgh, UK \& Royal Government of Bhutan, Bhutan, 604 pp.

Aitken, E., A.J.C. Grierson \& D.G. Long (1999). Family Primulaceae, pp. 515-568. In: Long, D.G. (eds.). Flora of Bhutan Including a Record of Plants from Sikkim and Darjeeling Volume 2 (Part 2). Royal Botanic Garden Edinburgh, UK \& Royal Government of Bhutan, Bhutan, 604 $\mathrm{pp}$

Champion, H.G. \& S.K. Seth (1968). A Revised Survey of the Forest Types of India. The Manager of Publications, Delhi.

Chase, M.W., M.J.M. Christenhusz, M.F. Fay, J.W. Byng, W.S. Judd, D.E. Soltis, D.J. Mabberley, A.N. Sennikov, P.S. Soltis \& P.F. Stevens (2016). An update of the Angiosperm Phylogeny Group classification for the orders and families of flowering plants: APG IV. Botanical Journal of the Linnean Society 181(1): 1-20. https://doi. org/10.1111/boj.12385

Dash, S.S. (2009). Traditional herbal remedies used in Sikkim, India. Nelumbo 51: 123-156. https://doi.org/10.20324/nelumbo/ v51/2009/58088

Dash, S.S. \& P. Singh (2011). Trees of Sikkim, pp. 95-138. In: Biodiversity of Sikkim-Exploring and Conserving a global Hotspot. Government of Sikkim, Gangtok.

Table 1. Detailed analysis of floristic diversity under various taxonomic groups (values in parentheses are percent contribution of the total).

\begin{tabular}{|c|c|c|c|c|c|c|c|c|c|}
\hline & \multicolumn{3}{|c|}{ Total Families } & \multicolumn{3}{|c|}{ Total Genera } & \multicolumn{3}{|c|}{ Total taxa } \\
\hline \multirow{2}{*}{ 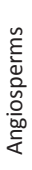 } & $\begin{array}{c}\text { Basal angiosperms } \\
\text { (Austrobaileyales } \\
\text { Takht. ex Reveal) }\end{array}$ & Mono cots & Eudicots & $\begin{array}{c}\text { Basal angiosperms } \\
\text { (Austrobaileyales } \\
\text { Takht. ex Reveal) }\end{array}$ & Monocots & Eudicots & $\begin{array}{c}\text { Basal angiosperms } \\
\text { (Austrobaileyales } \\
\text { Takht. ex Reveal) }\end{array}$ & Mono cots & Eudicots \\
\hline & 01 (1.85\%) & $\begin{array}{c}13 \\
(24.07 \%)\end{array}$ & $\begin{array}{c}40 \\
(74.07 \%)\end{array}$ & $\begin{array}{c}01 \\
(0.57 \%)\end{array}$ & $\begin{array}{c}30 \\
(17.34 \%)\end{array}$ & $\begin{array}{c}142 \\
(82.08 \%)\end{array}$ & $\begin{array}{c}01 \\
(0.24 \%)\end{array}$ & $\begin{array}{c}57 \\
(13.86 \%)\end{array}$ & $\begin{array}{c}353 \\
(85.88 \%)\end{array}$ \\
\hline $\begin{array}{l}\bar{\pi} \\
\stackrel{0}{0}\end{array}$ & \multicolumn{3}{|c|}{54} & \multicolumn{3}{|c|}{173} & \multicolumn{3}{|c|}{411} \\
\hline
\end{tabular}


Table 2. Enumeration of the flowering plants of Kyongnosla Alpine Sanctuary.

\begin{tabular}{|c|c|c|c|c|}
\hline Family & Scientific name of taxa & $\begin{array}{l}\text { *Growth } \\
\text { form }\end{array}$ & $\begin{array}{l}\text { Altitude } \\
(\mathrm{m})\end{array}$ & Collection number \& Herbarium acronym \\
\hline \multicolumn{5}{|c|}{ Basal angiosperms } \\
\hline Schisandraceae & $\begin{array}{l}\text { Schisandra grandiflora (Wall.) Hook.f. \& } \\
\text { Thomson }\end{array}$ & C & $3000-3600$ & S.S. Dash 24144 (BSHC) \\
\hline \multicolumn{5}{|c|}{ Monocots } \\
\hline \multirow{3}{*}{ Araceae } & Arisaema erubescens (Wall.) Schott & $\mathrm{H}$ & $2000-3400$ & S.S. Dash 22712 (BSHC) \\
\hline & Arisaema griffithii Schott & $\mathrm{H}$ & $2400-3700$ & S.S. Dash 27028 (BSHC); S. Lahiri 95710 (CAL) \\
\hline & Arisaema jacquemontii Blume & $\mathrm{H}$ & $2300-4200$ & S.S. Dash 22782 (BSHC); S. Lahiri 86682 (CAL) \\
\hline Tofieldiaceae & Tofieldia himalaica Baker & $\mathrm{H}$ & $3000-4500$ & B. Krishna 2068 (BSHC) \\
\hline \multirow{2}{*}{ Nartheciaceae } & Aletris glabra Bureau \& Franchet & $\mathrm{H}$ & $2400-4200$ & S.S. Dash 27247 (BSHC); S. Lahiri 86690 (CAL) \\
\hline & Aletris pauciflora (Klotzsch) Hand. -Mazz. & $\mathrm{H}$ & $2200-4900$ & S.S. Dash 22734 (BSHC); S. Lahiri 85719 (CAL) \\
\hline Smilacaceae & Smilax menispermoidea A.DC. & C & $2800-3600$ & $\begin{array}{l}\text { S.S. Dash 20995, } 24010 \text { (BSHC); S. Lahiri } \\
86685 \text { (CAL) }\end{array}$ \\
\hline \multirow{4}{*}{ Liliaceae } & Fritillaria cirrhosa D.Don & $\mathrm{H}$ & $3200-4500$ & S. Lahiri \& S.S. Dash $86714,64902,86623$ (CAL) \\
\hline & Gagea flavonutans (H. Hara) Zarrei \& Wilkin & $\mathrm{H}$ & $3800-4500$ & S. Lahiri \& S.S. Dash 85788 (CAL) \\
\hline & Lilium nanum Klotzsch & $\mathrm{H}$ & $3500-4500$ & S. Lahiri \& S.S. Dash 85784 (CAL) \\
\hline & Streptopus simplex D. Don & $\mathrm{H}$ & $3200-4300$ & S.S. Dash 22270 (BSHC); S. Lahiri 85701 (CAL) \\
\hline \multirow{11}{*}{ Orchidaceae } & $\begin{array}{l}\text { Bhutanthera albomarginata (King \& Pant.) } \\
\text { Renz }\end{array}$ & $\mathrm{H}$ & $3800-4300$ & S. Lahiri \& S.S. Dash 85753, 95737 (CAL) \\
\hline & Galearis spathulata (Lindl.) P.F.Hunt & $\mathrm{H}$ & $3000-4300$ & S. Lahiri \& S.S. Dash 86661 (CAL) \\
\hline & Gymnadenia orchidis Lindl. & $\mathrm{H}$ & $2800-4100$ & S. Lahiri \& S.S. Dash 85791 (CAL) \\
\hline & Herminium macrophyllum (D.Don) Dandy & $\mathrm{H}$ & $2400-4100$ & S.S. Dash 38383 (BSHC) \\
\hline & Herminium monorchis (L.) R.Br & $\mathrm{H}$ & $1200-4500$ & S. Lahiri \& S.S. Dash 95720 (CAL) \\
\hline & Platanthera exelliana Soó Hook.f & $\mathrm{H}$ & $3300-4500$ & S. Lahiri \& S.S. Dash 95721 (CAL). \\
\hline & Platanthera leptocaulon (Hook.f.) Soó & $\mathrm{H}$ & $3000-4000$ & S. Lahiri \& S.S. Dash 85705, 86695 (CAL). \\
\hline & Ponerorchis chusua (D.Don) Soó & $\mathrm{H}$ & $2500-4500$ & S. Lahiri \& S.S. Dash 86665, 85714(CAL) \\
\hline & Ponerorchis puberula (King \& Pantl.) Verm. & $\mathrm{H}$ & $2700-3600$ & S. Lahiri \& S.S. Dash 85702 (CAL) \\
\hline & Satyrium nepalense D.Don & $\mathrm{H}$ & $1800-4000$ & S. Lahiri \& S.S. Dash 86731(CAL) \\
\hline & $\begin{array}{l}\text { Satyrium nepalense var. ciliatum (Lindl.) } \\
\text { Hook.f. }\end{array}$ & $\mathrm{H}$ & $2500-4000$ & S. Lahiri \& S.S. Dash $86730,86747(\mathrm{CAL})$ \\
\hline Iridaceae & Iris clarkei Baker ex Hook.f. & $\mathrm{H}$ & $3300-4200$ & S.S. Dash 22702 (BSHC); S. Lahiri 85727 (CAL) \\
\hline \multirow[b]{2}{*}{ Amaryllidaceae } & Allium prattii C.H. Wright & $\mathrm{H}$ & $2500-4400$ & S. Lahiri \& S.S. Dash 85785, 95735 (CAL) \\
\hline & Allium wallichii Kunth & $\mathrm{H}$ & $3000-4500$ & $\begin{array}{l}\text { S.S. Dash 24071, 24122, } 27002 \text { (BSHC); S. Lahiri } \\
86737 \text { (CAL) }\end{array}$ \\
\hline \multirow{5}{*}{ Asparagaceae } & Maianthemum oleraceum (Baker) LaFrankie & $\mathrm{H}$ & $2700-3600$ & S. Lahiri \& S.S. Dash 95729 (CAL) \\
\hline & Maianthemum purpureum (Wall.) LaFrankie & $\mathrm{H}$ & $2700-4000$ & S. Lahiri \& S.S. Dash 86691, 86692 (CAL) \\
\hline & Polygonatum brevistylum Baker & $\mathrm{H}$ & $2000-3300$ & S.S. Dash 22710 (BSHC) \\
\hline & Polygonatum cirrhifolium (Wall.) Royle & $\mathrm{H}$ & $2200-4200$ & S.S. Dash 20992(a) (BSHC) \\
\hline & Polygonatum verticillatum (L.) All. & $\mathrm{H}$ & $2400-4300$ & $\begin{array}{l}\text { S.S. Dash 20992, } 22710 \text { (BSHC); S. Lahiri } 86694 \\
\text { (CAL) }\end{array}$ \\
\hline Eriocaulaceae & $\begin{array}{l}\text { Eriocaulon alpestre Hook.f. \& Thomson ex } \\
\text { Körn. }\end{array}$ & $\mathrm{H}$ & $2400-3900$ & S. Lahiri \& S.S. Dash 64907 (CAL) \\
\hline \multirow{7}{*}{ Juncaceae } & Juncus benghalensis Kunth & $\mathrm{H}$ & $2200-4200$ & S.S. Dash 22728 (BSHC); S. Lahiri 86696 (CAL) \\
\hline & Juncus bufonius L. & $\mathrm{H}$ & $2700-4500$ & S. Lahiri \& S.S. Dash 64934 (CAL) \\
\hline & Juncus cephalostigma Sam. & $\mathrm{H}$ & $3300-4500$ & S. Pradhan 6631 (BSHC) \\
\hline & Juncus chrysocarpus Buchenau & $\mathrm{H}$ & $3000-3600$ & D.C.S. Raju 3680 (BSHC) \\
\hline & Juncus concinnus D.Don & $\mathrm{H}$ & $2100-4300$ & S.S. Dash 27016 (BSHC) \\
\hline & Juncus effusus $\mathrm{L}$. & $\mathrm{H}$ & $2000-3400$ & S.S. Dash 27286 (BSHC) \\
\hline & Juncus grisebachii Buchenau & $\mathrm{H}$ & $2500-5000$ & S. Lahiri \& S.S. Dash 86745 (CAL) \\
\hline
\end{tabular}




\begin{tabular}{|c|c|c|c|c|}
\hline Family & Scientific name of taxa & $\begin{array}{l}\text { *Growth } \\
\text { form }\end{array}$ & $\begin{array}{l}\text { Altitude } \\
(\mathrm{m})\end{array}$ & Collection number \& Herbarium acronym \\
\hline \multirow{5}{*}{ Juncaceae } & Juncus himalensis Klotzsch & $\mathrm{H}$ & 2400-4200 & $\begin{array}{l}\text { S.S. Dash 24123,24130 (BSHC); S. Lahiri } 86657 \\
\text { (CAL) }\end{array}$ \\
\hline & Juncus kingii Rendle & $\mathrm{H}$ & $3700-4300$ & S.S. Dash 27231 (BSHC) \\
\hline & Juncus sikkimensis Hook.f. & $\mathrm{H}$ & $3600-4800$ & S.S. Dash 27289 \\
\hline & Juncus thomsonii Buchenau & $\mathrm{H}$ & $3600-3900$ & S.S. Dash 22208 (BSHC); S. Lahiri 64923 (CAL) \\
\hline & Luzula multiflora (Ehrh.) Lej. & $\mathrm{H}$ & $3000-4500$ & S.S. Dash 20996 (BSHC); S. Lahiri (CAL) \\
\hline \multirow{3}{*}{ Cyperaceae } & Carex gracilenta Boott ex Boeckeler & $\mathrm{H}$ & $3200-4300$ & S. Lahiri \& S.S. Dash 64909 (CAL) \\
\hline & Carex inanis Kunth & $\mathrm{H}$ & $1400-3700$ & S. Lahiri \& S.S. Dash 64929 (CAL) \\
\hline & Kobresia curticeps (C.B.Clarke) Kük. & $\mathrm{H}$ & 2500-4000 & G.P. Sinha \& D.G. Long 17748 (BSHC) \\
\hline \multirow{11}{*}{ Poaceae } & Agrostis inaequiglumis Griseb. & $\mathrm{H}$ & $3000-4500$ & S.S. Dash 24046 (BSHC); S. Lahiri 64911 (CAL) \\
\hline & Agrostis micrantha Steud. & $\mathrm{H}$ & $1600-3500$ & P. Singh \& S.K. Rai. 23784 (BSHC). \\
\hline & Agrostis nervosa Nees ex Trinius & $\mathrm{H}$ & $3300-4000$ & S.S. Dash 22789 (BSHC) \\
\hline & Calamagrostis emodensis Griseb. & $\mathrm{H}$ & 2200-4600 & S.S. Dash 24068 (BSHC) \\
\hline & Calamagrostis scabrescens Griseb. & $\mathrm{H}$ & $2000-3800$ & S. Lahiri \& S.S. Dash 64931 (CAL) \\
\hline & Cyathopus sikkimensis Stapf & $\mathrm{H}$ & $3600-4600$ & S.S. Dash 24105 (BSHC) \\
\hline & Deschampsia cespitosa (L.) P.Beauv. & $\mathrm{H}$ & $2700-4500$ & S.S. Dash 24104 (BSHC) \\
\hline & Festuca rubra L. & $\mathrm{H}$ & $3500-4200$ & S.S. Dash 27228 (BSHC) \\
\hline & Festuca valesiaca Schleicher ex Gaudin & $\mathrm{H}$ & $2500-3700$ & S.S. Dash 27044 (BSHC) \\
\hline & Poa pagophila Bor & $\mathrm{H}$ & $3600-5200$ & S.S. Dash 27035 (BSHC) \\
\hline & Poa pratensis $\mathrm{L}$. & $\mathrm{H}$ & $3000-5000$ & S.S. Dash 24113 (BSHC) \\
\hline \multicolumn{5}{|c|}{ Eudicots } \\
\hline \multirow{12}{*}{ Papaveraceae } & Corydalis cashmeriana Royle & $\mathrm{H}$ & $3000-5000$ & S. Lahiri \& S.S. Dash 85773 (CAL) \\
\hline & Corydalis filicina Prain & $\mathrm{H}$ & $2500-3500$ & S.S. Dash 27250 (BSHC); S. Lahiri 85774 (CAL) \\
\hline & Corydalis flaccida Hook.f. \& Thomson & $\mathrm{H}$ & $3300-4200$ & S.S. Dash 22738 (BSHC) \\
\hline & Corydalis juncea Wall. & $\mathrm{H}$ & $3600-4600$ & S.S. Dash 27226 (BSHC) \\
\hline & Corydalis meifolia Wall. & $\mathrm{H}$ & $3800-5200$ & S.S. Dash 27223 (BSHC); S. Lahiri 86612 (CAL) \\
\hline & Corydalis polygalina Hook.f. \& Thomson & $\mathrm{H}$ & $4000-5100$ & D.C.S. Raju 3655 (BSHC) \\
\hline & Corydalis pseudolongipes Lidén & $\mathrm{H}$ & $3000-4000$ & $\begin{array}{l}\text { S.S. Dash 24119,24018, } 27276 \text { (BSHC); S. Lahiri } \\
86634 \text { (CAL) }\end{array}$ \\
\hline & Corydalis sikkimensis (Prain) Fedde & $\mathrm{H}$ & $3000-4600$ & S.S. Dash 27006 (BSHC) \\
\hline & Corydalis trifoliata Franch. & $\mathrm{H}$ & $3600-4200$ & S.S. Dash 20977 (BSHC) \\
\hline & Meconopsis paniculata (D. Don) Prain & $\mathrm{H}$ & $3000-4200$ & S.S. Dash 22715 (BSHC); S. Lahiri 86659 (CAL) \\
\hline & Meconopsis simplicifolia (D. Don) Walp. & $\mathrm{H}$ & $3200-4500$ & S.S. Dash 20073 (BSHC); S. Lahiri 95705 (CAL) \\
\hline & Meconopsis villosa (Hook.f.) G.Taylor & $\mathrm{H}$ & 2700-4300 & S.S. Dash 22703(BSHC); S. Lahiri 85723(CAL) \\
\hline \multirow{4}{*}{ Berberidaceae } & Berberis angulosa Wall. ex Hook.f. \& Thomson & $\mathrm{S}$ & $3000-4000$ & $\begin{array}{l}\text { S.S. Dash } 27008 \text { (BSHC); S. Lahiri 85879, } 86651 \\
\text { (CAL) }\end{array}$ \\
\hline & Berberis griffithiana C.K.Schneid. & $\mathrm{H}$ & $2500-3300$ & S.S. Dash 22776 (BSHC) \\
\hline & Berberis macrosepala Hook.f \& Thomson & $\mathrm{H}$ & $3500-4400$ & S.S. Dash 22776(A) (BSHC) \\
\hline & Sinopodophyllum hexandrum (Royle) T.S.Ying & H & $2500-4500$ & S.S. Dash 27029 (BSHC). \\
\hline \multirow{8}{*}{ Ranunculaceae } & Aconitum dissectum D.Don & $\mathrm{H}$ & $3200-4500$ & S. Lahiri \& S.S. Dash 85739 (CAL) \\
\hline & Aconitum ferox Wallich ex Sering & $\mathrm{H}$ & $3000-5000$ & S.S. Dash 27225 (A) (BSHC); S. Lahiri 86754 (CAL) \\
\hline & Aconitum heterophylloides (Brühl) Stapf & $\mathrm{H}$ & $3300-4500$ & S. Lahiri \& S.S. Dash 86784 (CAL) \\
\hline & Aconitum novoluridum Munz & $\mathrm{H}$ & $2000-3500$ & S.S. Dash 27225(BSHC); S. Lahiri 64905 (CAL) \\
\hline & Aconitum palmatum D.Don & $\mathrm{H}$ & $2500-4000$ & S. Lahiri \& S.S. Dash 86795 (CAL) \\
\hline & Aconitum spicatum (Bruelh) Stapf & $\mathrm{H}$ & $3500-4000$ & S.S. Dash 27227 (BSHC); S. Lahiri 86783 (CAL) \\
\hline & Anemone obtusiloba D.Don & $\mathrm{H}$ & $2300-5300$ & S.S. Dash 22752 (BSHC) \\
\hline & Anemone rupestris Wallich ex Hook.f. & $\mathrm{H}$ & $2000-3500$ & S. Lahiri 95717 (CAL). \\
\hline
\end{tabular}




\begin{tabular}{|c|c|c|c|c|}
\hline Family & Scientific name of taxa & $\begin{array}{l}\text { *Growth } \\
\text { form }\end{array}$ & $\begin{array}{l}\text { Altitude } \\
(\mathrm{m})\end{array}$ & Collection number \& Herbarium acronym \\
\hline \multirow{18}{*}{ Ranunculaceae } & Anemone trullifolia Hook.f. \& Thomson & $\mathrm{H}$ & $2500-5000$ & S.S. Dash 27256 (BSHC) \\
\hline & Caltha palustris L. & $\mathrm{H}$ & $2000-3600$ & S.S. Dash 22721 (BSHC). \\
\hline & Caltha scaposa Hook.f. \& Thomson & $\mathrm{H}$ & $2500-4500$ & S.S. Dash 27225 (BSHC); S. Lahiri 86649 (CAL) \\
\hline & Clematis montana Buch. -Ham. ex DC. & $\mathrm{H}$ & $2000-4000$ & $\begin{array}{l}\text { S.S. Dash } 22701 \text { (BSHC); S. Lahiri 86788, } 95712 \\
\text { (CAL) }\end{array}$ \\
\hline & Delphinium candelabrum Ostenf. & $\mathrm{H}$ & $4100-5300$ & S.S. Dash 24189 (BSHC) \\
\hline & $\begin{array}{l}\text { Oxygraphis polypetala (Raf.) Hook.f. \& } \\
\text { Thomson }\end{array}$ & $\mathrm{H}$ & $3200-5300$ & S. Lahiri \& S.S. Dash 73248 (CAL) \\
\hline & Ranunculus brotherusii Freyn & $\mathrm{H}$ & $3000-3300$ & S.S. Dash 22747 (BSHC) \\
\hline & Ranunculus diffusus DC. & $\mathrm{H}$ & $1100-3100$ & S.S. Dash 22741 (BSHC) \\
\hline & Ranunculus ficariifolius Leveille \& Veniot & $\mathrm{H}$ & $1100-3200$ & S.S. Dash 27294 (BSHC) \\
\hline & Ranunculus hirtellus Royle ex D.Don & $\mathrm{H}$ & $3000-5000$ & Sinha \& Shukla 20550 (BSHC) \\
\hline & Ranunculus pulchellus C.A. Mey. & $\mathrm{H}$ & $2300-3200$ & S.S. Dash 20993, 20973 (BSHC) \\
\hline & Ranunculus trichophyllus Chaix & $\mathrm{H}$ & $3200-4100$ & Sinha \& Shukla 20550 (BSHC) \\
\hline & Thalictrum alpinum $\mathrm{L}$. & $\mathrm{H}$ & $2800-5000$ & S.S. Dash 19289 (BSHC); S. Lahiri 64924 (CAL) \\
\hline & Thalictrum chelidonii DC. & $\mathrm{H}$ & $2300-3600$ & S.S. Dash 24197 (BSHC) \\
\hline & Thalictrum elegans Wall. ex Royle & $\mathrm{H}$ & $2700-4200$ & S.S. Dash 24048 (BSHC) \\
\hline & Thalictrum javanicum Blume & $\mathrm{H}$ & $1500-3800$ & S.S. Dash 24196 (BSHC) \\
\hline & Thalictrum reniforme Wall. & $\mathrm{H}$ & $2800-3700$ & S. Lahiri \& S.S. Dash 85711 (CAL) \\
\hline & Thalictrum rutifolium Hook.f. \& Thomson & $\mathrm{H}$ & $2300-4300$ & S.K. Rai 9455 (BSHC) \\
\hline \multirow{4}{*}{ Grossulariaceae } & Ribes glaciale Wall & S & $2000-3500$ & S.S. Dash 27284 (BSHC) \\
\hline & Ribes laciniatum Hook.f. \& Thomson & S & $2400-3900$ & S.S. Dash 27206 (BSHC) \\
\hline & Ribes luridum Hook.f. \& Thomson & S & 2800-4200 & S.S. Dash 24150 (BSHC) \\
\hline & Ribes takare D.Don & S & $2700-4200$ & S.S. Dash 22796, 22799 (BSHC) \\
\hline \multirow{10}{*}{ Saxifragaceae } & Astilbe rivularis Buch.-Ham. ex D. Don & $\mathrm{H}$ & $2000-3600$ & $\begin{array}{l}\text { S.S. Dash 20976, } 24135 \text { (BSHC); S. Lahiri } 86662 \\
\text { (CAL) }\end{array}$ \\
\hline & Bergenia ciliata (Haw.) Sternb. & $\mathrm{H}$ & $2000-3500$ & S. Lahiri \& S.S. Dash 95753 (CAL) \\
\hline & $\begin{array}{l}\text { Bergenia purpurascens (Hook.f. \& Thomson) } \\
\text { Engl. }\end{array}$ & $\mathrm{H}$ & $3700-4800$ & S.S. Dash 27221 (BSHC); S. Lahiri 86718 (CAL) \\
\hline & Saxifraga assamensis Wadhwa & $\mathrm{H}$ & $3600-3900$ & S.S. Dash 24198 (BSHC) \\
\hline & Saxifraga brachypoda D.Don & $\mathrm{H}$ & $3000-5000$ & $\begin{array}{l}\text { S.S. Dash } 24198 \text { (BSHC); S. Lahiri 86775, } 85769 \\
\text { (CAL) }\end{array}$ \\
\hline & Saxifraga cordigera Hook.f. \& Thomson & $\mathrm{H}$ & $3000-4600$ & S.S. Dash 27208 (BSHC) \& S. Lahiri 86797 (CAL) \\
\hline & Saxifraga melanocentra Franch. & & $3000-4900$ & Sinha \& Shukla 20447 (BSHC) \\
\hline & Saxifraga moorcroftiana (Ser.) Wall. ex Sternb. & $\mathrm{H}$ & $2000-3500$ & $\begin{array}{l}\text { S.S. Dash 24126, } 27214 \text { (BSHC); S. Lahiri } 86776 \\
\text { (CAL) }\end{array}$ \\
\hline & Saxifraga pallida Wall. ex Ser. & $\mathrm{H}$ & $3000-5000$ & S. Lahiri \& S.S. Dash 86617 (CAL) \\
\hline & Saxifraga parnassifolia D.Don & $\mathrm{H}$ & $2000-3500$ & S.S. Dash 24001 (BSHC); S. Lahiri 86786 (CAL) \\
\hline \multirow{7}{*}{ Crassulaceae } & $\begin{array}{l}\text { Rhodiola bupleuroides (Wall. ex Hook.f. \& } \\
\text { Thomson) S.H.Fu }\end{array}$ & $\mathrm{H}$ & $3400-5700$ & $\begin{array}{l}\text { S.S. Dash 27274(BSHC); S. Lahiri 85730, } 85757 \\
\text { (CAL) }\end{array}$ \\
\hline & Rhodiola chrysanthemifolia (H. Lév.) S.H.Fu & $\mathrm{H}$ & $3200-4200$ & S.S. Dash 19275 (BSHC) \\
\hline & Rhodiola cretinii (Raymond-Hamet) H.Ohba & $\mathrm{H}$ & $3700-4400$ & S. Lahiri \& S.S. Dash 85752 (CAL) \\
\hline & $\begin{array}{l}\text { Rhodiola crenulata (Hook.f. \& Thomson) } \\
\text { H.Ohba }\end{array}$ & $\mathrm{H}$ & $2800-5600$ & S.S. Dash 27248 (BSHC). \\
\hline & $\begin{array}{l}\text { Rhodiola fastigiata (Hook. f. \& Thomson) } \\
\text { S.H.Fu }\end{array}$ & $\mathrm{H}$ & $3500-5400$ & S. Lahiri \& S.S. Dash 74489 (CAL) \\
\hline & Rhodiola himalensis (D. Don) S.H.Fu & $\mathrm{H}$ & $3600-4200$ & $\begin{array}{l}\text { S.S. Dash 6620, } 85752 \text { (BSHC); S. Lahiri } 74416 \\
\text { (CAL). }\end{array}$ \\
\hline & Rhodiola wallichiana (Hook.) S.H.Fu & $\mathrm{H}$ & $3500-3800$ & $\begin{array}{l}\text { S.S. Dash 27255, 24012; S. Lahiri 86715, } 86748 \\
\text { (CAL) }\end{array}$ \\
\hline
\end{tabular}




\begin{tabular}{|c|c|c|c|c|}
\hline Family & Scientific name of taxa & $\begin{array}{l}\text { *Growth } \\
\text { form }\end{array}$ & $\begin{array}{l}\text { Altitude } \\
(\mathrm{m})\end{array}$ & Collection number \& Herbarium acronym \\
\hline \multirow{2}{*}{$\begin{array}{l}\text { Fabaceae } \\
\text { (= Leguminosae) }\end{array}$} & Astragalus sikkimensis Bunge & $\mathrm{H}$ & $2600-4500$ & S. Lahiri \& S.S. Dash 64922 (CAL) \\
\hline & Parochetus communis D.Don & $\mathrm{H}$ & $1800-3500$ & S. Lahiri \& S.S. Dash 86654 (CAL) \\
\hline \multirow{22}{*}{ Rosaceae } & Fragaria nubicola (Lindl. ex Hook.f.) Lacaita & $\mathrm{H}$ & $2500-3900$ & S.S. Dash 24045 (BSHC); S. Lahiri 86728 (CAL) \\
\hline & Fragaria vesca $\mathrm{L}$. & $\mathrm{H}$ & $2000-3200$ & S. Singh 3398 (BSHC) \\
\hline & Potentilla eriocarpa Wall. ex Lehm. & $\mathrm{H}$ & $2700-5000$ & S.S. Dash 22753 (BSHC) \\
\hline & $\begin{array}{l}\text { Potentilla fruticosa var. arbuscula (D. Don) } \\
\text { Maxim. }\end{array}$ & $\mathrm{s}$ & $2600-4600$ & S. Lahiri \& S.S. Dash 85742 (CAL) \\
\hline & Potentilla leuconota D.Don & $\mathrm{H}$ & $2200-4600$ & S. Lahiri \& S.S. Dash 86640 (CAL) \\
\hline & Potentilla peduncularis D.Don & $\mathrm{H}$ & $3000-4800$ & S.S. Dash 22735 (BSHC); S. Lahiri 95754 (CAL) \\
\hline & Potentilla polyphylla Wall. ex Lehm. & $\mathrm{H}$ & 2500-4500 & S.S. Dash 22707 (BSHC) \\
\hline & Potentilla saundersiana Royle & $\mathrm{H}$ & $2600-5200$ & S.S. Dash 22762 (BSHC) \\
\hline & Prunus carmesina H.Hara & $\mathrm{T}$ & $1900-3000$ & S.S. Dash \& S. Lahiri 95760 (CAL) \\
\hline & Prunus cornuata & $\mathrm{T}$ & $2000-3200$ & S.S. Dash \& S. Lahiri 95761 (CAL) \\
\hline & Rosa sericea Wall. ex Lindl. & $\mathrm{s}$ & $3000-4000$ & $\begin{array}{l}\text { S.S. Dash 222705, 24036, 27246, } 27041 \text { (BSHC); } \\
\text { S. Lahiri } 86627 \text { (CAL) }\end{array}$ \\
\hline & Rubus lasiostylus Focke & $\mathrm{s}$ & $2700-3200$ & S. Lahiri \& S.S. Dash 85717 (CAL) \\
\hline & Rubus mesogaeus Focke & $\mathrm{s}$ & $2700-3600$ & S.S. Dash 24194 (BSHC) \\
\hline & Rubus pectinarioides H.Hara & S & $2000-3500$ & D.C.S. Raju \& S. Singh 3661 (BSHC) \\
\hline & Rubus pedunculosus D.Don & $\mathrm{s}$ & $2600-3700$ & S.S. Dash 22784 (BSHC) \\
\hline & Rubus thomsonii Focke & $\mathrm{s}$ & $2700-3400$ & A.K. Sahoo 3588 (BSHC) \\
\hline & Sibbaldia parviflora Willd. & $\mathrm{H}$ & $2000-3500$ & S. Lahiri \& S.S. Dash 86723 (CAL) \\
\hline & Sorbus foliolosa (Wall.) Spach & $\mathrm{T}$ & $2500-4200$ & S.S. Dash 20987 (BSHC); S. Lahiri 86780 (CAL) \\
\hline & Sorbus prattii Koehne & $\mathrm{T}$ & $2000-4500$ & S.S. Dash 24133 (BSHC) \\
\hline & Sorbus microphylla (Wall. ex Hook.f.) Wenz. & $\mathrm{T}$ & $3200-4200$ & S.S. Dash 24038 (BSHC) \\
\hline & Spiraea arcuata Hook.f. & s & $2700-4000$ & S.S. Dash 22794 (BSHC) \\
\hline & Spiraea canescens D.Don & $\mathrm{S}$ & $2700-4000$ & S.S. Dash 24154 (BSHC); S. Lahiri 95739 (CAL) \\
\hline Betulaceae & Betula utilis D.Don & $\mathrm{T}$ & $2600-4200$ & S.S. Dash 22751, 24019 (BSHC) \\
\hline \multirow{5}{*}{ Celastraceae } & Euonymus tingens Wall & $\mathrm{H}$ & $1300-3700$ & S.S. Dash 24049 (BSHC) \\
\hline & Parnassia chinensis Franch. & $\mathrm{H}$ & $2500-4400$ & $\begin{array}{l}\text { S.S. Dash } 27208 \text { (BSHC); S. Lahiri 86733, 86632, } \\
86638 \text { (CAL) }\end{array}$ \\
\hline & Parnassia cooperi W.E.Evans & $\mathrm{H}$ & $3000-3500$ & S.S. Dash 22741(BSHC) \\
\hline & Parnassia nubicola Wall. ex Royle & $\mathrm{H}$ & $3200-4350$ & $\begin{array}{l}\text { S.S. Dash 24131,27203 (BSHC); S. Lahiri } \\
86733 \text { (CAL) }\end{array}$ \\
\hline & Parnassia tenella Hook.f. \& Thomson & $\mathrm{H}$ & $2800-3400$ & S.S. Dash 22741(BSHC) \\
\hline Oxalidaceae & Oxalis leucolepis Diels & $\mathrm{H}$ & 2800-4000 & S. Lahiri \& S.S. Dash 86664 (CAL) \\
\hline \multirow{3}{*}{ Hypericaceae } & Hypericum choisianum Wall. ex N.Robson & $\mathrm{H}$ & 2100-4000 & S.S. Dash 20980 (BSHC) \\
\hline & Hypericum hookerianum Wight \& Arn. & $\mathrm{H}$ & $1900-3400$ & S.S. Dash 24077 (BSHC); S. Lahiri 86724 (CAL) \\
\hline & $\begin{array}{l}\text { Hypericum petiolulatum Hook.f. \& Thomson } \\
\text { ex Dyer }\end{array}$ & $\mathrm{H}$ & $1000-3100$ & S. Lahiri \& S.S. Dash 86653 (CAL) \\
\hline Violaceae & Viola biflora L. & $\mathrm{H}$ & 2500-4300 & S.S. Dash 22733 (BSHC); S. Lahiri 85772 (CAL) \\
\hline \multirow{5}{*}{ Salicaceae } & Salix calyculata Hook.f. ex Andersson & $\mathrm{S}$ & $3400-4700$ & S.S. Dash 22749 (BSHC); S. Lahiri 74463 (CAL) \\
\hline & Salix daltoniana Andersson & $\mathrm{S}$ & $3000-4400$ & S.S. Dash 24013 (BSHC) \\
\hline & Salix longiflora Wall. ex Andersson & S & $500-4000$ & Sinha \& Shukla 20470 (BSHC) \\
\hline & Salix serpyllum Anderson & s & $3200-4500$ & S.S. Dash 24050 (BSHC); S. Lahiri 85878 (CAL). \\
\hline & Salix sikkimensis Andersson & $\mathrm{s}$ & $3350-4000$ & S.S. Dash 27290 (BSHC); S. Lahiri 86631 (CAL) \\
\hline Euphorbiaceae & Euphorbia sikkimensis Boiss. & $\mathrm{H}$ & $2500-3500$ & S. Lahiri \& S.S. Dash 85766 (CAL) \\
\hline
\end{tabular}




\begin{tabular}{|c|c|c|c|c|}
\hline Family & Scientific name of taxa & $\begin{array}{l}\text { *Growth } \\
\text { form }\end{array}$ & $\begin{array}{c}\text { Altitude } \\
(\mathrm{m})\end{array}$ & Collection number $\&$ Herbarium acronym \\
\hline \multirow{3}{*}{ Geraniaceae } & Geranium donianum Sweet & $\mathrm{H}$ & $2400-4800$ & S.S. Dash 24043 (BSHC); S. Lahiri 74455 (CAL) \\
\hline & Geranium nepalense Sweet & $\mathrm{H}$ & $1000-3600$ & $\begin{array}{l}\text { S.S. Dash 24040, } 22757 \text { (BSHC); S. Lahiri } 85721 \\
\text { (CAL) }\end{array}$ \\
\hline & Geranium polyanthes Edgew. \& Hook.f. & $\mathrm{H}$ & 2900-4000 & S.S. Dash 24065 (BSHC) \\
\hline \multirow{6}{*}{ Onagraceae } & $\begin{array}{l}\text { Circaea alpina subsp. imaicola (Asch. \& } \\
\text { Magnus) Kitam. }\end{array}$ & $\mathrm{H}$ & $2000-4000$ & B. Krishna 6431 (BSHC) \\
\hline & Epilobium amurense Hausskn. & $\mathrm{H}$ & $2100-4000$ & S. Lahiri \& S.S. Dash 86633 (CAL) \\
\hline & Epilobium sikkimense Hausskn. & $\mathrm{H}$ & $2000-3750$ & S. Lahiri \& S.S. Dash 85741 (CAL) \\
\hline & Epilobium tibetanum Hausskn. & $\mathrm{H}$ & $2000-4500$ & Sinha \& Shukla 21598 (BSHC) \\
\hline & Epilobium wallichianum Hausskn. & $\mathrm{H}$ & $2200-4100$ & Sinha \& Shukla 21594 (BSHC) \\
\hline & Epilobium williamsii P.H.Raven & $\mathrm{H}$ & $2300-4700$ & S. Lahiri \& S.S. Dash 74422 (CAL). \\
\hline \multirow{2}{*}{ Sapindaceae } & Acer acuminatum Wall. ex D.Don & $\mathrm{T}$ & $3300-4700$ & S. Lahiri \& S.S. Dash 95744 (CAL) \\
\hline & Acer campbellii Hook.f. \& Thomson ex Hiern & $\mathrm{T}$ & $2000-3500$ & S. Lahiri \& S.S. Dash 85708 (CAL) \\
\hline \multirow{8}{*}{$\begin{array}{l}\text { Brassicaceae } \\
\text { (= Cruciferae) }\end{array}$} & $\begin{array}{l}\text { Arcyosperma primulifolium (Thomson) } \\
\text { O.E.Schulz }\end{array}$ & $\mathrm{H}$ & $3000-4500$ & Sinha \& Shukla 20539 (BSHC) \\
\hline & Barbarea elata Hook.f. \& Thomson & $\mathrm{H}$ & $3200-3800$ & S.S. Dash 22749 (A) (BSHC) \\
\hline & Barbarea intermedia Boreau & $\mathrm{H}$ & $2800-4200$ & S.S. Dash 22779 (BSHC); S. Lahiri 95716 (CAL) \\
\hline & Cardamine griffithi Hook.f., Thomson & $\mathrm{H}$ & 2000-3800 & S.S. Dash 22739 (BSHC) \\
\hline & Cardamine macrophylla Willd. & $\mathrm{H}$ & $2500-4200$ & $\begin{array}{l}\text { S.S. Dash 22754, } 27053 \text { (BSHC); S. Lahiri 86628, } \\
85877 \text { (CAL) }\end{array}$ \\
\hline & $\begin{array}{l}\text { Cardamine pulchella (Hook.f. \& Thomson) Al- } \\
\text { Shehbaz \& G.Yang }\end{array}$ & $\mathrm{H}$ & $3000-4100$ & S.S. Dash 22766 (BSHC) S. Lahiri 85716 (CAL) \\
\hline & Pegaeophyton minutum H.Hara & $\mathrm{H}$ & $3700-4200$ & S. Lahiri 95749 (CAL) \\
\hline & Thlaspi montanum L. & $\mathrm{H}$ & $1500-3000$ & S. Pradhan 6608 (BSHC) \\
\hline \multirow{12}{*}{ Polygonaceae } & Aconogonon campanulatum (Hook.f.) H.Hara & H & $3000-3600$ & $\begin{array}{l}\text { S.S. Dash 20736, 24035, } 86642 \text { (BSHC); S. Lahiri } \\
86642 \text { (CAL) }\end{array}$ \\
\hline & $\begin{array}{l}\text { Aconogonon campanulatum var. oblongum } \\
\text { (Meisn.) H.Hara }\end{array}$ & H & $3000-3600$ & S.S. Dash 22717 (BSHC). \\
\hline & Aconogonon molle (D. Don) H.Hara & $\mathrm{H}$ & 2000-4000 & S.S. Dash 24081 (BSHC); S. Lahiri 86620 (CAL) \\
\hline & $\begin{array}{l}\text { Aconogonon polystachyum (Wall. ex Meisn.) } \\
\text { M.Král }\end{array}$ & $\mathrm{H}$ & 2000-4000 & $\begin{array}{l}\text { S.S. Dash 20735, 27242, } 24005 \text { (BSHC); S. Lahiri } \\
86742 \text { (CAL) }\end{array}$ \\
\hline & Aconogonon rude (Meisn.) S.S.Dash \& P.Singh & $\mathrm{H}$ & 2000-3300 & S.S. Dash 24138 (BSHC) \\
\hline & Bistorta affinis (D.Don) Greene & $\mathrm{H}$ & $3500-5000$ & S.S. Dash 27293 (BSHC); S. Lahiri 74426 (CAL) \\
\hline & Bistorta amplexicaulis (D.Don) Green & $\mathrm{H}$ & $3000-4200$ & $\begin{array}{l}\text { S.S. Dash 20999, } 24002 \text { (BSH); S. Lahiri } 85713 \\
\text { (CAL) }\end{array}$ \\
\hline & Bistorta emodi (Meisn.) H.Hara & $\mathrm{H}$ & $3200-4000$ & S.S. Dash 27253 (BSHC); S. Lahiri 64935 (CAL) \\
\hline & Bistorta macrophylla (D.Don) Soják & $\mathrm{H}$ & $3500-4200$ & S.S. Dash 27251 (BSHC) \\
\hline & $\begin{array}{l}\text { Bistorta suffulta (Maximowicz) Greene ex. } \\
\text { H.Gross }\end{array}$ & $\mathrm{H}$ & $3000-4200$ & S. Kumar \& P. Singh 11640 (BSHC) \\
\hline & Bistorta vacciniifolia (Wall. ex Meisn.) Greene & $\mathrm{H}$ & 2600-3900 & S.S. Dash 24109 (BSHC); S. Lahiri 86758 (CAL) \\
\hline & Bistorta vivipara (L) S.F.Gray & $\mathrm{H}$ & $2200-3850$ & $\begin{array}{l}\text { S.S. Dash } 22719 \text { (BSHC); S. Lahiri 86726, } 85800 \\
\text { (CAL) }\end{array}$ \\
\hline \multirow{8}{*}{ Polygonaceae } & Koenigia delicatula (Meisn.) H.Hara & $\mathrm{H}$ & $2600-5000$ & $\begin{array}{l}\text { S.S. Dash 18593, 24060, } 27240 \text { (BSHC); S. Lahiri } \\
74492,85720 \text { (CAL) }\end{array}$ \\
\hline & Koenigia forrestii (Diels) Mesicek \& Soják & $\mathrm{H}$ & $2800-5200$ & D.G. Long et al. 18245 (BSHC) \\
\hline & Koenigia nepalensis D.Don & $\mathrm{H}$ & $2800-5000$ & $\begin{array}{l}\text { S.S. Dash } 27032 \text { (BSHC); S. Lahiri 85720, } 85738 \\
\text { (CAL) }\end{array}$ \\
\hline & Koenigia nepalensis D.Don var. villosa & $\mathrm{H}$ & $3000-4900$ & S.S. Dash 24041(BSHC) \\
\hline & Koenigia pilosa Maxim. & $\mathrm{H}$ & $3000-4500$ & Sinha \& Shukla 21565 (BSHC) \\
\hline & Oxyria digyna (L.) Hill & $\mathrm{H}$ & $3000-5500$ & $\begin{array}{l}\text { S.S. Dash 18532, } 27283 \text { (BSHC); S. Lahiri } 86619 \\
\text { (CAL). }\end{array}$ \\
\hline & Persicaria nepalensis (Meisn.) Miyabe & $\mathrm{H}$ & 2500-3900 & $\begin{array}{l}\text { S.S. Dash 27032,24041(BSHC); S. Lahiri 86738, } \\
86739,85797 \text { (CAL) }\end{array}$ \\
\hline & $\begin{array}{l}\text { Persicaria runcinata (Buch.-Ham. ex D.Don) } \\
\text { H. Gross }\end{array}$ & H & $1000-3800$ & $\begin{array}{l}\text { S.S. Dash } 24032 \text { (BSHC); S. Lahiri 86735, 85710, } \\
85711 \text { (CAL) }\end{array}$ \\
\hline
\end{tabular}




\begin{tabular}{|c|c|c|c|c|}
\hline Family & Scientific name of taxa & $\begin{array}{l}\text { *Growth } \\
\text { form }\end{array}$ & $\begin{array}{l}\text { Altitude } \\
(\mathrm{m})\end{array}$ & Collection number \& Herbarium acronym \\
\hline \multirow{4}{*}{ Polygonaceae } & Rheum acuminatum Hook.f. \& Thomson & $\mathrm{H}$ & $3000-4100$ & S.S. Dash 18569 (BSHC); S. Lahiri 85735 (CAL). \\
\hline & Rheum nobile Hook.f. \& Thomson & $\mathrm{H}$ & $3000-5200$ & S.S. Dash 24124 (BSHC). \\
\hline & Rumex dentatus L. & $\mathrm{H}$ & $2200-3800$ & S.S. Dash 20985 (BSHC) \\
\hline & Rumex nepalensis Spreng. & $\mathrm{H}$ & $1500-3600$ & S.S. Dash 22775, 24066, 27268 (BSHC) \\
\hline \multirow{7}{*}{ Caryophyllaceae } & Arenaria debilis Hook.f & $\mathrm{H}$ & $3000-4900$ & S. Lahiri \& S.S. Dash 85726 (CAL) \\
\hline & Cerastium glomeratum Thuill. & $\mathrm{H}$ & $1000-4200$ & S.S. Dash 27287 (BSHC); S. Lahiri 86625 (CAL) \\
\hline & Gypsophila cerastoides D.Don & $\mathrm{H}$ & $3000-4200$ & $\begin{array}{l}\text { S.S. Dash 22763,22787 (BSHC); S. Lahiri } 85764 \\
\text { (CAL) }\end{array}$ \\
\hline & Silene caespitella F.Williams & $\mathrm{H}$ & $2500-5100$ & S.S. Dash 24190 (A) (BSHC); S. Lahiri 86727 (CAL) \\
\hline & Silene indica (Roxb.) Roxb. ex Otth & $\mathrm{H}$ & $2500-5000$ & B. Krishna \& B. Mitra 6414 (BSHC) \\
\hline & Silene nigrescens (Edgew.) Majumdar & $\mathrm{H}$ & $3000-4500$ & S. Lahiri \& S.S. Dash 64903 (CAL) \\
\hline & Stellaria subumbellata Edgew. & H & $3500-5300$ & S.S. Dash 24118 (BSHC) \\
\hline \multirow{10}{*}{ Balsaminaceae } & Impatiens bicornuta Wall. & $\mathrm{H}$ & $2400-3200$ & P.K. Hajra 514 (BSHC) \\
\hline & Impatiens cymbifera Hook.f. & $\mathrm{H}$ & $2100-3200$ & S. Lahiri \& S.S. Dash 95746 (CAL) \\
\hline & Impatiens falcifer Hook.f. & $\mathrm{H}$ & $2400-3800$ & S. Lahiri \& S.S. Dash 95747 (CAL) \\
\hline & Impatiens jurpia Buch.-Ham. & $\mathrm{H}$ & $2000-3000$ & S.S. Dash 20988 (BSHC) \\
\hline & Impatiens kingii Hook.f. & $\mathrm{H}$ & $2800-4000$ & S. Lahiri \& S.S. Dash 86708 (CAL) \\
\hline & Impatiens racemosa DC. & H & $1500-3300$ & S.S. Dash 27207(BSHC); S. Lahiri 95748 (CAL) \\
\hline & Impatiens radiata Hook.f. & $\mathrm{H}$ & $2000-3600$ & S. Lahiri \& S.S. Dash 86639, 86641(CAL) \\
\hline & Impatiens serratifolia Hook.f. & $\mathrm{H}$ & $2400-3300$ & S. Lahiri \& S.S. Dash 85712,86643 (CAL) \\
\hline & Impatiens spirifer Hook.f. \& Thomson & $\mathrm{H}$ & $1200-3200$ & S.S. Dash 27257 (BSHC) \\
\hline & Impatiens urticifolia Wall. & $\mathrm{H}$ & $2300-3400$ & S. Lahiri \& S.S. Dash 86707 (CAL) \\
\hline \multirow{17}{*}{ Primulaceae } & Androsace croftii Watt & $\mathrm{H}$ & $3000-4000$ & S.S. Dash \& S. Lahiri 95762 (CAL) \\
\hline & Primula calderiana Balf.f. \& Cooper & $\mathrm{H}$ & $3500-4500$ & Sinha \& Shukla 21589 (BSHC) \\
\hline & Primula capitata Hook.f & $\mathrm{H}$ & $3500-4500$ & S.S. Dash 24092 (BSHC); S. Lahiri 85775 (CAL) \\
\hline & Primula drummondiana Craib & $\mathrm{H}$ & $3000-4200$ & S. Lahiri \& S.S. Dash 74545 (CAL) \\
\hline & Primula elongata Watt & $\mathrm{H}$ & $3800-4300$ & S.S. Dash \& S. Lahiri 95759 (CAL) \\
\hline & Primula glomerata Pax & $\mathrm{H}$ & $3300-5700$ & S. Lahiri \& S.S. Dash 85775 (CAL) \\
\hline & Primula involucrata Wall. ex Duby & $\mathrm{H}$ & $3000-4500$ & S. Lahiri \& S.S. Dash 85750 (CAL) \\
\hline & Primula kingii Watt & $\mathrm{H}$ & $3500-4300$ & S. Lahiri \& S.S. Dash 85728 (CAL) \\
\hline & Primula minutissima Jacquem. ex Duby & $\mathrm{H}$ & $3700-5200$ & S.S. Dash 27280 (BSHC) \\
\hline & Primula obliqua W.W.Sm. & $\mathrm{H}$ & $3500-4500$ & S. Lahiri \& S.S. Dash 86614(CAL) \\
\hline & Primula primulina (Spreng.) H.Hara & $\mathrm{H}$ & $3400-5000$ & S. Lahiri \& S.S. Dash 85780, 85781 (CAL) \\
\hline & Primula reticulata Wall. & $\mathrm{H}$ & $3000-4000$ & S. Pradhan 6603 (BSHC) \\
\hline & Primula sapphirina Hook.f. \& Thomson ex Watt & $\mathrm{H}$ & $3500-4800$ & S. Lahiri \& S.S. Dash 85796 (CAL) \\
\hline & Primula scapigera (Hook.f.) Craib & $\mathrm{H}$ & $2000-4000$ & S. Lahiri \& S.S. Dash 73245 (BSHC) \\
\hline & Primula sikkimensis Hook.f. & $\mathrm{H}$ & $3200-4400$ & $\begin{array}{l}\text { S.S. Dash 22722, 27212(BSHC); S. Lahiri } 85707 \\
\text { (CAL) }\end{array}$ \\
\hline & Primula vaginata Watt & $\mathrm{H}$ & $2500-4500$ & S. Lahiri \& S.S. Dash 95726 (CAL) \\
\hline & Primula wattii King ex Watt & $\mathrm{H}$ & $4000-4600$ & S. Lahiri \& S.S. Dash 95704 (CAL) \\
\hline \multirow{6}{*}{ Ericaceae } & Cassiope fastigiata (Wall.) D.Don & $\mathrm{H}$ & $3000-4500$ & S. Lahiri \& S.S. Dash 85787 (CAL) \\
\hline & Cassiope selaginoides Hook.f. \& Thomson & $\mathrm{H}$ & $3000-4200$ & S. S. Dash 24149 (BSHC); S. Lahiri 95715 (CAL) \\
\hline & Enkianthus deflexus (Griff.) C.K. Schneid. & $\mathrm{s}$ & $2000-3600$ & S. S. Dash 24142 (BSHC) \\
\hline & Gaultheria griffithiana Wight & $\mathrm{H}$ & 2100-4000 & P. Singh \& S.K. Rai 22692 (BSHC) \\
\hline & Gaultheria nummularioides D.Don & $\mathrm{H}$ & $1800-3500$ & S.S. Dash 24045(BSHC); S. Lahiri 86729 (CAL) \\
\hline & Gaultheria semi-infera (C.B. Clarke) Airy Shaw & $\mathrm{S}$ & $2100-3000$ & S.S. Dash 27208 (BSHC); S. Lahiri 85745 (CAL) \\
\hline
\end{tabular}




\begin{tabular}{|c|c|c|c|c|}
\hline Family & Scientific name of taxa & $\begin{array}{l}\text { *Growth } \\
\text { form }\end{array}$ & $\begin{array}{l}\text { Altitude } \\
(\mathrm{m})\end{array}$ & Collection number \& Herbarium acronym \\
\hline \multirow{22}{*}{ Ericaceae } & Gaultheria trichophylla Royle & $\mathrm{H}$ & $3000-3900$ & $\begin{array}{l}\text { S.S. Dash 27039, 22768, 27235, } 24045 \text { (BSHC); S. } \\
\text { Lahiri } 73247 \text { (CAL) }\end{array}$ \\
\hline & Lyonia ovalifolia (Wall.) Drude & $\mathrm{T}$ & $1500-3400$ & S. Lahiri \& S.S. Dash 64901, 85745 (CAL) \\
\hline & $\begin{array}{l}\text { Lyonia villosa (Wall. ex C.B.Clarke) Hand. - } \\
\text { Mazz. }\end{array}$ & $\mathrm{T}$ & $3000-3900$ & S.S. Dash 22746 (BSHC); S. Lahiri 64920 (CAL) \\
\hline & Rhododendron anthopogon D.Don & $\mathrm{s}$ & $2400-4900$ & S.S. Dash 27291 (BSHC); S. Lahiri 95751 (CAL) \\
\hline & Rhododendron arboreum Sm. & $\mathrm{T}$ & $1500-3800$ & S. Lahiri \& S.S. Dash 73242 (CAL) \\
\hline & Rhododendron baileyi Balf.f. & $\mathrm{S}$ & $3000-4000$ & S. S. Dash 24151 (BSHC) \\
\hline & Rhododendron camelliiflorum Hook.f. & $\mathrm{S}$ & $2500-3700$ & S.S. Dash 24146 (BSHC) \\
\hline & Rhododendron campanulatum D.Don & $\mathrm{T}$ & 2800-4000 & S. Lahiri \& S.S. Dash 73244 (CAL) \\
\hline & $\begin{array}{l}\text { Rhododendron campanulatum subsp. } \\
\text { aeruginosum (Hook. f.) D.F.Chamb. }\end{array}$ & $\mathrm{T}$ & $3500-4600$ & $\begin{array}{l}\text { S. S. Dash } 24145 \text { (BSHC); } \\
\text { S. Lahiri } 73249 \text { (CAL) }\end{array}$ \\
\hline & Rhododendron campylocarpum Hook.f. & $\mathrm{T}$ & $3000-4600$ & S. Lahiri \& S.S. Dash 95752 CAL) \\
\hline & Rhododendron cinnabarinum Hook.f. & $\mathrm{T}$ & $2400-4000$ & S.S. Dash 24147 (BSHC) \\
\hline & Rhododendron fulgens Hook.f. & $S$ & $3700-4500$ & S.S. Dash 22723 (BSHC); S. Lahiri 73251(CAL) \\
\hline & Rhododendron glaucophyllum Rehder & $\mathrm{S}$ & $2700-3700$ & $\begin{array}{l}\text { S.S. Dash 24070, } 24146 \text { (BSHC); S. Lahiri } 73240 \\
\text { (CAL). }\end{array}$ \\
\hline & Rhododendron grande Wight & $\mathrm{T}$ & $2500-3200$ & S.S. Dash 27219 (BSHC) \\
\hline & Rhododendron griffithianum Wight & $\mathrm{T}$ & 2000-3000 & P.K. Hajra 2215 (BSHC) \\
\hline & Rhododendron hodgsonii Hook.f. & $\mathrm{T}$ & $3000-3600$ & S. Lahiri \& S.S. Dash 73255 (CAL) \\
\hline & Rhododendron lanatum Hook.f. & $\mathrm{T}$ & $3000-4000$ & S.S. Dash 24143 (BSHC) \\
\hline & Rhododendron lepidotum Wall. ex G.Don & $\mathrm{S}$ & $3000-4500$ & $\begin{array}{l}\text { S.S. Dash 24063, } 22723 \text { (BSHC); S. Lahiri } 73252 \\
\text { (CAL) }\end{array}$ \\
\hline & Rhododendron niveum Hook.f. & $\mathrm{S}$ & $2900-3700$ & P. Singh \& B.P. Uniyal 16228 (BSHC) \\
\hline & Rhododendron setosum D.Don & $S$ & $3000-4500$ & $\begin{array}{l}\text { P.K. Hajra } 308 \text { (BSHC); S. Lahiri \& S.S. Dash } 86711 \\
\text { CAL) }\end{array}$ \\
\hline & Rhododendron thomsonii Hook.f. & $\mathrm{T}$ & $3000-4200$ & S.S. Dash 22785 (BSHC); S. Lahiri 73243 (CAL) \\
\hline & $\begin{array}{l}\text { Vaccinium nummularia Hook.f. \& Thomson ex } \\
\text { C.B.Clarke }\end{array}$ & $\mathrm{E}$ & $2440-3200$ & P.K. Hajra 341, B. Krishna 6366 (BSHC) \\
\hline \multirow{3}{*}{ Rubiaceae } & Galium acutum Edgew. & $\mathrm{H}$ & 2000-4100 & S. Lahiri \& S.S. Dash 74434 (CAL) \\
\hline & Galium asperifolium Wall. & $\mathrm{H}$ & $400-3500$ & S.S. Dash \& S. Lahiri 95758 (CAL) \\
\hline & Galium rebae R.R.Mill & $\mathrm{H}$ & 2000-4000 & S. Lahiri \& S.S. Dash 86636 (CAL) \\
\hline \multirow{14}{*}{ Gentianaceae } & Crawfurdia puberula C.B.Clarke & $\mathrm{H}$ & $2700-3200$ & S.S. Dash 24003 (BSHC) \\
\hline & Gentiana bryoides Burkill & $\mathrm{H}$ & $3000-4500$ & S. Lahiri \& S.S. Dash 73253 (CAL) \\
\hline & Gentiana capitata Buch.-Ham. ex D.Don & $\mathrm{H}$ & $2000-3500$ & S.S. Dash 24030 (a) (BSHC) \\
\hline & Gentiana crassuloides Bureau \& Franch. & $\mathrm{H}$ & $3600-4600$ & S.S. Dash 22771 (BSHC) \\
\hline & Gentiana elwesii C.B.Clarke & $\mathrm{H}$ & $3600-4500$ & S.S. Dash 27281 (BSHC); S. Lahiri 86716 (CAL) \\
\hline & Gentiana leucomelaena Maxim. & $\mathrm{H}$ & $3000-5000$ & S. Lahiri \& S.S. Dash 85754 (CAL) \\
\hline & Gentiana prolata Balf.f. & $\mathrm{H}$ & $3000-4500$ & S. Lahiri \& S.S. Dash 74402 (CAL) \\
\hline & Gentiana recurvata C.B.Clarke & $\mathrm{H}$ & $3000-4300$ & S. Lahiri \& S.S. Dash 86676 (CAL) \\
\hline & $\begin{array}{l}\text { Gentiana recurvata subsp. prainii (Burkill) } \\
\text { Halda }\end{array}$ & $\mathrm{H}$ & $3000-4300$ & S. Lahiri \& S.S. Dash 85718 (CAL) \\
\hline & Gentiana sikkimensis C.B.Clarke & $\mathrm{H}$ & $2700-5200$ & $\begin{array}{l}\text { S.S. Dash 24067, } 27949 \text { (BSHC); S. Lahiri } 86772 \\
\text { (CAL) }\end{array}$ \\
\hline & Gentiana stylophora C.B.Clarke & $\mathrm{H}$ & $3000-4500$ & $\begin{array}{l}\text { S.S. Dash 27005, } 6636 \text { (BSHC); S. Lahiri } 85760 \\
\text { (CAL) }\end{array}$ \\
\hline & Gentiana tubiflora (G. Don) Griseb. & $\mathrm{H}$ & $4000-5300$ & $\begin{array}{l}\text { S.S. Dash } 24029 \text { (BSHC); S. Lahiri 86670, 86755, } \\
86622 \text { (CAL) }\end{array}$ \\
\hline & Halenia elliptica D.Don & H & $2000-4200$ & $\begin{array}{l}\text { S.S. Dash 27271, } 24030 \text { (BSHC); S. Lahiri 86740, } \\
86644 \text { (CAL) }\end{array}$ \\
\hline & $\begin{array}{l}\text { Lomatogonium brachyantherum (C.B. Clarke) } \\
\text { Fernald }\end{array}$ & $\mathrm{H}$ & $3800-4300$ & S. Lahiri \& S.S. Dash 86762 (CAL) \\
\hline
\end{tabular}




\begin{tabular}{|c|c|c|c|c|}
\hline Family & Scientific name of taxa & $\begin{array}{l}\text { *Growth } \\
\text { form }\end{array}$ & $\begin{array}{l}\text { Altitude } \\
(\mathrm{m})\end{array}$ & Collection number \& Herbarium acronym \\
\hline \multirow{6}{*}{ Gentianaceae } & $\begin{array}{l}\text { Omphalogramma elwesianum (King ex Watt) } \\
\text { Franch. }\end{array}$ & $\mathrm{H}$ & $3300-4200$ & P.K. Hajra 344(BSHC). \\
\hline & $\begin{array}{l}\text { Swertia bimaculata (Siebold \& Zucc.) Hook.f. \& } \\
\text { Thomson ex C.B.Clarke }\end{array}$ & $\mathrm{H}$ & $2000-3500$ & S.S. Dash 24047 (BSHC); S. Lahiri 74453 (CAL) \\
\hline & Swertia hookeri C.B.Clarke & $\mathrm{H}$ & $3700-4500$ & S. Lahiri \& S.S. Dash 85756, 95736 (CAL) \\
\hline & Swertia multicaulis D.Don & $\mathrm{H}$ & $3600-4700$ & S. Lahiri \& S.S. Dash 74451 (CAL). \\
\hline & Swertia teres (G.Don) J.Shah & $\mathrm{H}$ & $3000-4500$ & S. Lahiri \& S.S. Dash 74414, 86744 (CAL) \\
\hline & Veratrilla burkilliana (W.W.Sm.) Harry Sm. & $\mathrm{H}$ & $3200-4600$ & S. Lahiri \& S. S. Dash 95702 (CAL). \\
\hline \multirow{4}{*}{ Boraginaceae } & Cynoglossum wallichii G.Don & $\mathrm{H}$ & $1300-3600$ & S.S. Dash 27274 (BSHC); S. Lahiri 85715 (CAL) \\
\hline & $\begin{array}{l}\text { Cynoglossum wallichii var. glochidiatum (Wall. } \\
\text { ex Benth.) Kazmi }\end{array}$ & $\mathrm{H}$ & $1300-3600$ & S. Lahiri \& S.S. Dash 85737 (CAL) \\
\hline & Hackelia uncinata (Benth.) C.E.C.Fisch. & H & $3300-4200$ & S.S. Dash 22758 (BSHC); S. Lahiri 86675 (CAL) \\
\hline & Maharanga emodi (Wall.) A.DC. & $\mathrm{H}$ & $2500-3300$ & $\begin{array}{l}\text { S.S. Dash 22751, } 24019 \text { (BSHC); S. Lahiri } 85732 \\
\text { (CAL) }\end{array}$ \\
\hline Carlemanniaceae & Carlemannia griffithii Benth. & H & $1000-2900$ & S.S. Dash 13892 (BSHC) \\
\hline Calceolariaceae & Calceolaria tripartita Ruiz \& Pav. & $\mathrm{H}$ & $1900-5000$ & S. Lahiri \& S.S. Dash 64936 (CAL) \\
\hline \multirow{6}{*}{ Plantaginaceae } & Lagotis kunawurensis Rupr. & $\mathrm{H}$ & $2700-5600$ & S. Lahiri \& S.S. Dash 85761 (CAL) \\
\hline & $\begin{array}{l}\text { Neopicrorhiza scrophulariiflora (Pennell) } \\
\text { D.Y.Hong }\end{array}$ & $\mathrm{H}$ & $3600-4400$ & S. Lahiri \& S.S. Dash 86700 (BSHC) \\
\hline & Plantago depressa Willd. & $\mathrm{H}$ & $2600-3900$ & S. Lahiri \& S.S. Dash 86725 (BSHC) \\
\hline & Veronica cana Wall. ex Benth. & $\mathrm{H}$ & $2743-4115$ & $\begin{array}{l}\text { S.S. Dash 22736, 27043, } 24089 \text { (BSHC); S. Lahiri } \\
86669 \text { (CAL) }\end{array}$ \\
\hline & $\begin{array}{l}\text { Veronica szechuanica subsp. sikkimensis } \\
\text { (Hook.f.) D.Y.Hong }\end{array}$ & H & $2900-4200$ & S. Lahiri \& S.S. Dash 86673 (CAL) \\
\hline & Hemiphragma heterophyllum Wall. & $\mathrm{H}$ & 2600-4100 & S.S. Dash 24026 (BSHC); S. Lahiri 73249 (CAL). \\
\hline \multirow{2}{*}{ Scrophulariaceae } & Scrophularia elatior Wall. ex Benth. & H & $2000-3200$ & S.S. Dash 22998 (BSHC) \\
\hline & Euphrasia bhutanica Pugsley & $\mathrm{H}$ & $3000-4200$ & S. Lahiri \& S.S. Dash 64904, 85771 (CAL) \\
\hline Acanthaceae & Strobilanthes wallichii Nees & $\mathrm{H}$ & $2500-3700$ & S. Lahiri \& S. S. Dash 85740, 86635 (CAL) \\
\hline \multirow{3}{*}{ Lentibulariaceae. } & Utricularia brachiata Oliver & $\mathrm{H}$ & 2500-4200 & S. Lahiri \& S.S. Dash 86698 (CAL) \\
\hline & Utricularia christopheri P.Taylor & $\mathrm{H}$ & 2100-3900 & S. Lahiri \& S.S. Dash 86768 (CAL) \\
\hline & Utricularia multicaulis Oliv. & $\mathrm{H}$ & $3050-4115$ & S. Lahiri \& S.S. Dash 85734 (CAL) \\
\hline \multirow{11}{*}{ Lamiaceae } & Ajuga integrifolia Buch.-Ham. & $\mathrm{H}$ & $1200-2900$ & S.S. Dash 22785 (BSHC) \\
\hline & Elsholtzia blanda (Benth.) Benth. & $\mathrm{H}$ & $1000-3000$ & S.S. Dash 27233, 27288 (BSHC) \\
\hline & Elsholtzia densa Benth. & $\mathrm{H}$ & $2500-3600$ & S.S. Dash 24116 (BSHC) \\
\hline & Elsholtzia strobilifera (Benth.) Benth. & $\mathrm{H}$ & 2300-4000 & $\begin{array}{l}\text { S.S. Dash } 24969 \text { (BSHC); S. Lahiri 86759, } 86756 \\
\text { (CAL) }\end{array}$ \\
\hline & Paraphlomis brevifolia C.Y.Wu \& H.W.Li & $\mathrm{H}$ & $2000-3200$ & S. Lahiri \& S.S. Dash 85706 (CAL) \\
\hline & $\begin{array}{l}\text { Phlomoides tibetica (C.Marquand \& A.Shaw) } \\
\text { Kamelin \& Makhm. }\end{array}$ & $\mathrm{H}$ & $2500-4700$ & S.S. Dash 22767(BSHC) \\
\hline & Plectranthus mollis (Aiton) Spreng. & $\mathrm{H}$ & $1000-2900$ & P.K. Hajra 518 (BSHC) \\
\hline & Prunella vulgaris $\mathrm{L}$. & $\mathrm{H}$ & 2600-4200 & S. Lahiri 86697 (CAL) \\
\hline & Salvia campanulata Wall. ex Benth. & $\mathrm{H}$ & 2200-4000 & $\begin{array}{l}\text { S.S. Dash 24102,24048 (BSHC); S. Lahiri } 86674 \\
\text { (CAL). }\end{array}$ \\
\hline & Salvia nubicola Wall. ex Sweet & $\mathrm{H}$ & $2600-3700$ & S. Lahiri \& S.S. Dash 95727 (CAL) \\
\hline & Salvia roborowskii Maxim. & $\mathrm{H}$ & $2500-3700$ & S. Lahiri \& S.S. Dash 85767 (CAL) \\
\hline \multirow{5}{*}{ Orobanchaceae } & Pedicularis denudata Hook.f. & $\mathrm{H}$ & $3500-4500$ & S. Lahiri \& S.S. Dash 86717 (CAL) \\
\hline & Pedicularis elwesii Hook.f. & $\mathrm{H}$ & $3200-4600$ & S.S. Dash 27037 (BSHC) \\
\hline & Pedicularis flexuosa Hook.f. & $\mathrm{H}$ & 2800-4000 & S. Lahiri \& S.S. Dash 86712 (CAL) \\
\hline & Pedicularis furfuracea Wall. ex Benth. & $\mathrm{H}$ & $3500-4000$ & S.S. Dash 22783 (BSHC); S. Lahiri 85731 (CAL) \\
\hline & $\begin{array}{l}\text { Pedicularis gracilis subsp. macrocarpa (Prain) } \\
\text { Tsoong }\end{array}$ & $\mathrm{H}$ & $2900-3600$ & S. Lahiri \& S.S. Dash 85724 (CAL). \\
\hline
\end{tabular}




\begin{tabular}{|c|c|c|c|c|}
\hline Family & Scientific name of taxa & $\begin{array}{l}\text { *Growth } \\
\text { form }\end{array}$ & $\begin{array}{l}\text { Altitude } \\
\text { (m) }\end{array}$ & Collection number \& Herbarium acronym \\
\hline \multirow{10}{*}{ Orobanchaceae } & $\begin{array}{l}\text { Pedicularis longiflora var. tubiformis (Klotzsch) } \\
\text { Tsoong }\end{array}$ & $\mathrm{H}$ & $3000-5000$ & S.S. Dash 24017 (BSHC); S. Lahiri 86656 (CAL). \\
\hline & Pedicularis megalantha D. Don & $\mathrm{H}$ & $2800-5000$ & S.S. Dash 27210 (BSHC); S. Lahiri 86752 (CAL) \\
\hline & Pedicularis microcalyx Hook.f. & $\mathrm{H}$ & $3200-4500$ & S. Lahiri \& S.S. Dash 85786 (CAL) \\
\hline & Pedicularis mollis Wall. ex Benth. & $\mathrm{H}$ & $2700-4200$ & S. Lahiri \& S.S. Dash 85798 (CAL) \\
\hline & Pedicularis porrecta Wall. & $\mathrm{H}$ & $3300-5000$ & S. Lahiri \& S.S. Dash 86630 (CAL) \\
\hline & Pedicularis rhinanthoides Schrenk & $\mathrm{H}$ & $2500-5500$ & S.S. Dash 22774 (BSHC) \\
\hline & Pedicularis roylei Maxim. & $\mathrm{H}$ & $3400-5500$ & S. Lahiri \& S.S. Dash 85789 (CAL) \\
\hline & Pedicularis siphonantha D.Don & $\mathrm{H}$ & $3200-4500$ & S. Lahiri \& S.S. Dash 86629 (CAL) \\
\hline & Pedicularis tenuicaulis Prain & $\mathrm{H}$ & $3500-4000$ & S.S. Dash 27249 (BSHC) \\
\hline & Pedicularis wallichii Bunge & $\mathrm{H}$ & $3600-5000$ & S. Lahiri \& S.S. Dash 95734 (CAL) \\
\hline \multirow{10}{*}{ Campanulaceae } & Campanula pallida Wall. & $\mathrm{H}$ & $1200-3500$ & S.S. Dash 24200 (BSHC) \\
\hline & Codonopsis benthamii Hook.f. \& Thomson & $\mathrm{H}$ & 2100-4300 & S. Lahiri \& S.S. Dash 86684 (CAL) \\
\hline & Codonopsis foetens Hook.f. \& Thomson & $\mathrm{H}$ & $3600-4500$ & S. Lahiri \& S.S. Dash 85747 (CAL) \\
\hline & Codonopsis subsimplex Hook.f. \& Thomson & $\mathrm{H}$ & $3000-4200$ & S. Lahiri \& S.S. Dash $86751,85743,86637$ (CAL) \\
\hline & Codonopsis thalictrifolia Wall. & $\mathrm{H}$ & $3400-5000$ & S. Lahiri \& S.S. Dash 95750 (CAL) \\
\hline & Cyananthus incanus Hook.f. \& Thomson & $\mathrm{H}$ & $3500-4600$ & S. Lahiri \& S.S. Dash 86736 (CAL) \\
\hline & Cyananthus inflatus Hook.f. \& Thomson & $\mathrm{H}$ & 2700-4800 & S. Lahiri \& S.S. Dash 86774 (CAL) \\
\hline & Cyananthus lobatus Wall. ex Benth. & $\mathrm{H}$ & $3300-4200$ & S. Lahiri \& S.S. Dash $86777,85755,85799$ (CAL) \\
\hline & Cyananthus pedunculatus C.B.Clarke & $\mathrm{H}$ & $3000-4500$ & S. Lahiri \& S.S. Dash 86785 (CAL) \\
\hline & Lobelia erectiuscula H.Hara & $\mathrm{H}$ & $2200-4200$ & S. Lahiri \& S.S. Dash 85709 (CAL) \\
\hline \multirow{21}{*}{$\begin{array}{l}\text { Asteraceae } \\
\text { (= Compositae) }\end{array}$} & Ainsliaea aptera DC. & $\mathrm{H}$ & $3000-3600$ & S.S. Dash 24101 (BSHC) \\
\hline & Anaphalis contorta (D. Don) Hook.f. & $\mathrm{H}$ & $2000-5000$ & S. Lahiri \& S.S. Dash 64921(CAL) \\
\hline & Anaphalis margaritacea (L.) Benth. \& Hook.f. & $\mathrm{H}$ & 2000-4000 & S. Lahiri \& S.S. Dash 95743 (CAL) \\
\hline & $\begin{array}{l}\text { Anaphalis nepalensis (Spreng.) Hand. -Mazz. } \\
\text { Var. nepalensis }\end{array}$ & H & $2800-5200$ & S. Lahiri \& S.S. Dash 86789 (CAL) \\
\hline & $\begin{array}{l}\text { Anaphalis nepalensis var. monocephala (DC.) } \\
\text { Hand.-Mazz. }\end{array}$ & $\mathrm{H}$ & $2800-5200$ & S. Lahiri \& S.S. Dash 86770 (CAL) \\
\hline & Anaphalis triplinervis (Sims) Sims ex C.B. Clarke & $\mathrm{H}$ & $1500-4200$ & $\begin{array}{l}\text { S.S. Dash } 24020 \text { (BSHC); S. Lahiri 86650, 86741, } \\
86778 \text { (CAL) }\end{array}$ \\
\hline & $\begin{array}{l}\text { Anaphalis triplinervis var. monocephala (DC.) } \\
\text { Airy Shaw }\end{array}$ & $\mathrm{H}$ & $1500-4200$ & $\begin{array}{l}\text { S.S. Dash 22724,27273 (BSHC); S. Lahiri } 85733 \text {, } \\
86650 \text { (CAL) }\end{array}$ \\
\hline & Artemisia nilagirica (C.B.Clarke) Pamp. & $\mathrm{H}$ & $1500-3300$ & S.S. Dash 24039 (BSHC) \\
\hline & Aster flaccidus Bunge & $\mathrm{H}$ & $3800-5000$ & S.S. Dash 20457 (BSHC); S. Lahiri (CAL) \\
\hline & Aster himalaicus C.B.Clarke & $\mathrm{H}$ & $2500-5000$ & $\begin{array}{l}\text { S.S. Dash 27034, } 24024 \text { (BSHC); S. Lahiri } 85748 \\
\text { (CAL) }\end{array}$ \\
\hline & Aster tricephalus C.B.Clarke & $\mathrm{H}$ & $3000-4000$ & S. Lahiri \& S.S. Dash 86753 (CAL) \\
\hline & $\begin{array}{l}\text { Cacalia mortonii (C.B.Clarke) Kitam. ex H. } \\
\text { Koyama }\end{array}$ & $\mathrm{H}$ & $2500-3800$ & S. Lahiri \& S.S. Dash 85759 (CAL) \\
\hline & Carpesium cernuum L. & $\mathrm{H}$ & 2900-3500 & S.S. Dash \& S.S. Lahiri 95757 (CAL). \\
\hline & Chaetoseris cyanea (D.Don) C.Shih & $\mathrm{H}$ & $2600-4000$ & S. Lahiri \& S.S. Dash 85704 (CAL) \\
\hline & Chaetoseris macrantha (C.B.Clarke) C.Shih & $\mathrm{H}$ & $3000-5000$ & S.S. Dash 27267 (BSHC) \\
\hline & Cirsium falconeri (Hook.f.) Petr. & $\mathrm{H}$ & $3000-4200$ & $\begin{array}{l}\text { S.S. Dash 20998, } 24014 \text { (BSHC); S. Lahiri } 86767 \\
\text { (CAL) }\end{array}$ \\
\hline & Cremanthodium oblongatum C.B.Clarke & $\mathrm{H}$ & $3600-5300$ & S.S. Dash 27277 (BSHC); S. Lahiri 85783 (CAL) \\
\hline & Cremanthodium reniforme (DC.) Benth. & $\mathrm{H}$ & $3300-4500$ & S.S. Dash 27279 (BSHC); S. Lahiri 85765 (CAL) \\
\hline & Dubyaea hispida (D.Don) DC. & $\mathrm{H}$ & $3000-4500$ & $\begin{array}{l}\text { S.S. Dash } 24120 \text { (BSHC); S. Lahiri 86648, } 86734 \\
\text { (CAL) }\end{array}$ \\
\hline & Himalaiella deltoidea (DC.) Raab-Straube & $\mathrm{H}$ & $1000-3400$ & D.C.S. Raju, 5050 (BSHC) \\
\hline & Jurinea cooperi J.Anthony & $\mathrm{H}$ & $3500-4300$ & S.S. Dash 24112 (BSHC) \\
\hline
\end{tabular}




\begin{tabular}{|c|c|c|c|c|}
\hline Family & Scientific name of taxa & $\begin{array}{l}\text { *Growth } \\
\text { form }\end{array}$ & $\begin{array}{c}\text { Altitude } \\
(\mathrm{m})\end{array}$ & Collection number \& Herbarium acronym \\
\hline \multirow{23}{*}{$\begin{array}{l}\text { Asteraceae } \\
\text { (= Compositae) }\end{array}$} & Jurinea dolomiaea Boiss. & $\mathrm{H}$ & $3500-4300$ & S.S. Dash 27285 (BSHC) \\
\hline & Lactuca brunoniana (DC.) Wall. ex C.B.Clarke & $\mathrm{H}$ & $3300-3800$ & S.S. Dash 24078 (BSHC) \\
\hline & $\begin{array}{l}\text { Lactuca lessertiana (Wall. ex DC.) Wall. ex } \\
\text { C.B.Clarke }\end{array}$ & $\mathrm{H}$ & $3400-4100$ & S.S. Dash 24125 (BSHC); S. Lahiri 86787 (CAL) \\
\hline & Laphangium luteoalbum (L.) Tzvelev & $\mathrm{H}$ & $2000-3800$ & S.S. Dash 27241(BSHC); S. Lahiri 74535 (CAL) \\
\hline & $\begin{array}{l}\text { Leontopodium nivale subsp. alpinum (Cass.) } \\
\text { Greuter }\end{array}$ & $\mathrm{H}$ & $3500-4500$ & S. Lahiri \& S.S. Dash 85777 (CAL) \\
\hline & Ligularia amplexicaulis DC. & $\mathrm{H}$ & $3200-4400$ & S.S. Dash 24037 (BSHC); S. Lahiri 86671 (CAL) \\
\hline & Ligularia fischeri (Ledeb.) Turcz. & $\mathrm{H}$ & $2800-3600$ & S. Lahiri \& S.S. Dash 85762 (CAL) \\
\hline & Ligularia kingiana (W.W.Smith) R.Mathur & $\mathrm{H}$ & $3500-4000$ & S.S. Dash 22062, 27030 (BSHC) \\
\hline & $\begin{array}{l}\text { Ligularia virgaurea (Maxim.) Mattf. ex Rehder } \\
\text { \& Kobuski }\end{array}$ & $\mathrm{H}$ & $2400-4700$ & S. Lahiri \& S.S. Dash 85768 (CAL) \\
\hline & Myriactis nepalensis Less. & $\mathrm{H}$ & $700-3700$ & S. Lahiri \& S.S. Dash 64919 (CAL) \\
\hline & Saussurea gossypiphora D.Don & $\mathrm{H}$ & $3500-4500$ & S.S. Dash 27217 (BSHC) \\
\hline & Saussurea nepalensis Spreng. & $\mathrm{H}$ & $3200-5000$ & $\begin{array}{l}\text { S.S. Dash } 24110,27262 \text { (BSHC) S. Lahiri } 86773, \\
86798,85792 \text { (CAL) }\end{array}$ \\
\hline & Saussurea obvallata (DC.) Edgew. & $\mathrm{H}$ & $3500-4500$ & S.S. Dash 27265(BSHC); S. Lahiri 85782(CAL) \\
\hline & Saussurea roylei (DC.) Sch.-Bip & $\mathrm{H}$ & $3500-4500$ & S.S. Dash 27261 (BSHC) \\
\hline & Saussurea piptathera Edgew. & $\mathrm{H}$ & $3200-4200$ & S. Lahiri \& S.S. Dash 95745 (CAL) \\
\hline & Saussurea sughoo C.B. Clarke & $\mathrm{H}$ & $3500-4800$ & B. Krishna \& S. Singh 3100 (BSHC) \\
\hline & Saussurea uniflora (DC.) Wall. ex Sch.Bip. & $\mathrm{H}$ & $3000-4700$ & S. Lahiri \& S.S. Dash 86771 (CAL) \\
\hline & Senecio graciliflorus (Wall.) DC. & $\mathrm{H}$ & $3500-4600$ & $\begin{array}{l}\text { S.S. Dash 24111, 27031(a) (BSHC); S. Lahiri } \\
85776 \text { (CAL) }\end{array}$ \\
\hline & Senecio raphanifolius Wallich ex DC. & $\mathrm{H}$ & $2800-4400$ & $\begin{array}{l}\text { S.S. Dash } 22714,24016,27042 \text { (BSHC); S. Lahiri } \\
86699,85703 \text { (CAL) }\end{array}$ \\
\hline & Synotis alata C.Jeffrey \& Y.L.Chen & $\mathrm{H}$ & 2000-4000 & S. Lahiri \& S.S. Dash 86681, 64932 (CAL) \\
\hline & Tanacetum atkinsonii (C.B.Clarke) Kitam & $\mathrm{H}$ & $2000-4200$ & S. Lahiri \& S.S. Dash 85794 (CAL) \\
\hline & Taraxacum eriopodum (D.Don) DC. & $\mathrm{H}$ & 2000-4500 & S. Lahiri \& S.S. Dash 86647, 86750, 64918 (CAL) \\
\hline & $\begin{array}{l}\text { Tibetoseris depressa (Hook.f. \& Thomson) } \\
\text { Sennikov }\end{array}$ & $\mathrm{H}$ & $3600-5000$ & S. Lahiri \& S.S. Dash 86779 (CAL) \\
\hline \multirow{7}{*}{ Caprifoliaceae } & $\begin{array}{l}\text { Dipsacus atratus Hook.f. \& Thomson ex } \\
\text { C.B.Clarke }\end{array}$ & $\mathrm{H}$ & $3000-4000$ & S. Lahiri \& S.S. Dash 86757 (BSHC) \\
\hline & Lonicera lanceolata Wall. & $\mathrm{s}$ & $2440-3880$ & S.S. Dash 20986,24134 (BSHC) \\
\hline & Morina nepalensis D.Don & $\mathrm{H}$ & $3000-4800$ & S. Lahiri \& S.S. Dash 86618 (CAL) \\
\hline & Nardostachys jatamansi (D.Don) DC. & $\mathrm{H}$ & $2800-5000$ & S. Lahiri \& S.S. Dash 85880,85750 (CAL) \\
\hline & Triplostegia glandulifera Wall. ex DC. & $\mathrm{H}$ & 2000-4000 & S. Lahiri \& S.S. Dash 86763 (CAL) \\
\hline & Valeriana hardwickii Wall. & $\mathrm{H}$ & $1200-3800$ & S. Lahiri \& S.S. Dash 64926, 85790 (CAL) \\
\hline & Viburnum erubescens Wall. & $\mathrm{S}$ & $2500-3500$ & S. Lahiri \& S.S. Dash 86800 (CAL) \\
\hline \multirow{4}{*}{ Araliaceae } & Aralia apioides Hand.-Mazz. & $\mathrm{H}$ & $2700-3600$ & S. Lahiri \& S.S. Dash 86679 (CAL) \\
\hline & Aralia tibetana G.Hoo & $\mathrm{H}$ & $3200-3500$ & S.S. Dash 20990,24052,24192 (BSHC) \\
\hline & Panax bipinnatifidus Seem. & $\mathrm{H}$ & $2500-3500$ & S.S. Dash 22727 (BSHC) \\
\hline & Panax pseudoginseng Wall. & $\mathrm{H}$ & $1500-3600$ & S. Lahiri \& S.S. Dash 86644, 86764, 64925 (CAL) \\
\hline \multirow{7}{*}{ Apiaceae } & Angelica cyclocarpa (C.Norman) M.Hiroe & $\mathrm{S}$ & 2800-4000 & S. Lahiri \& S.S. Dash 86613 (CAL) \\
\hline & Bupleurum candollei Wall. ex DC. & $\mathrm{H}$ & $2000-3500$ & S.S. Dash 27010 (BSHC) \\
\hline & $\begin{array}{l}\text { Bupleurum dalhousianum (C.B.Clarke) Koso- } \\
\text { Pol. }\end{array}$ & $\mathrm{H}$ & 2000-4800 & S.S. Dash 22780 (BSHC); S. Lahiri 95728 (CAL) \\
\hline & Cortiella hookeri (C.B.Clarke) C.Norman & $\mathrm{H}$ & $4000-5000$ & S.S. Dash 27274 (BSHC) \\
\hline & Heracleum wallichii DC. & $\mathrm{H}$ & $3000-3700$ & S.S. Dash 27245 (BSHC) \\
\hline & Oenanthe thomsonii C.B.Clarke & $\mathrm{H}$ & $1500-3200$ & S.S. Dash 24051 (BSHC); S. Lahiri 95744 (CAL) \\
\hline & Pleurospermum apiolens C.B.Clarke & $\mathrm{H}$ & $3200-5000$ & S. Lahiri \& S.S. Dash 86621 (CAL) \\
\hline
\end{tabular}




\begin{tabular}{|c|c|c|c|c|}
\hline Family & Scientific name of taxa & $\begin{array}{l}\text { *Growth } \\
\text { form }\end{array}$ & $\begin{array}{l}\text { Altitude } \\
(\mathrm{m})\end{array}$ & Collection number \& Herbarium acronym \\
\hline \multirow{5}{*}{ Apiaceae } & Pleurospermum hookeri C.B.Clarke & $\mathrm{H}$ & $3500-4200$ & S.S. Dash \& S.S. Lahiri 95756 (CAL) \\
\hline & $\begin{array}{l}\text { Pleurospermopsis sikkimensis (C.B.Clarke) } \\
\text { C.Norman }\end{array}$ & $\mathrm{H}$ & $3700-4500$ & S.S. Dash 27001 (BSHC); S. Lahiri 86782 (CAL) \\
\hline & Sanicula elata Buch.-Ham. ex D.Don & $\mathrm{H}$ & $1000-3200$ & S.S. Dash 24100 (BSHC) \\
\hline & $\begin{array}{l}\text { Selinum wallichianum (DC.) Raizada \& } \\
\text { H.O.Saxena }\end{array}$ & $\mathrm{H}$ & $3400-4000$ & S.S. Dash 27269 (BSHC); S. Lahiri, 85729 (CAL) \\
\hline & Vicatia coniifolia Wall. ex DC. & $\mathrm{H}$ & $3500-4200$ & S.S. Dash 27011 (BSHC); S. Lahiri 86672 (CAL) \\
\hline \multicolumn{5}{|c|}{ Gymnosperm } \\
\hline \multirow{2}{*}{ Cupressaceae } & Juniperus recurva Buch.-Ham. ex D.Don & $\mathrm{T}$ & $1800-3900$ & $\begin{array}{l}\text { S.S. Dash 24004, } 22769 \text { (BSHC); S. Lahiri } 86693 \\
\text { (CAL) }\end{array}$ \\
\hline & Juniperus squamata f. wilsonii Rehder & S & $1600-4500$ & S. Lahiri \& S.S. Dash 74471 (CAL) \\
\hline \multirow{3}{*}{ Pinaceae } & Abies densa Griff. & $\mathrm{T}$ & $2800-3700$ & $\begin{array}{l}\text { S.S. Dash 24034,16210 (A) (BSHC); S. Lahiri } \\
86615 \text { (CAL) }\end{array}$ \\
\hline & Larix griffithii Hook.f. & $\mathrm{T}$ & $3000-4100$ & S.S. Dash 24148 (BSHC) \\
\hline & Picea spinulosa (Griff.) A. Henry & $\mathrm{T}$ & 2900-3600 & S.S. Dash 24152 (BSHC) \\
\hline
\end{tabular}

*-Growth form | C-Climber | E-Epiphyte | H-Herb | S-Shrub | T-Tree.

Dash, S.S., S. Lahiri, A. Ghosh \& B.K. Sinha (2020). Notes on two lesser known Codonopsis (Campanulaceae) from eastern Himalaya, India. Rheedea 30(2): 286-292. https://doi.org/10.22244/ rheedea.2020.30.02.05

Gogoi, R., S. Borah, S.S. Dash, \& P. Singh (2018). Balsams of Eastern Himalaya - A Regional Revision. Botanical Survey of India, Kolkata, 256 pp.

Grierson, A.J.C. \& D.G. Long (1983). Family Polygonaceae, pp. 153175. In: Flora of Bhutan Including a Record of Plants from Sikkim Volume 1 (Part 1). Royal Botanic Garden, Edinburgh, 186 pp.

Grierson, A.J.C. \& D.G. Long (1987). Family Rosaceae, pp. 529-606. In Flora of Bhutan Including a Record of Plants from Sikkim volume 1 (Part 3). Royal Botanic Garden, Edinburgh, 366 pp.

Grierson, A.J.C. \& L.S. Springate (2001). Family Compositae (Asteraceae), pp. 1397-1632. In: Springate, L.S. (eds.). Flora of Bhutan Including a Record of Plants from Sikkim and Darjeeling Volume 2 (Part 3). Royal Botanic Garden Edinburgh, UK \& Royal Government of Bhutan, Bhutan, 639 pp.

Grierson, A.J.C. (1984). Family Ranunculaceae, pp. 283-320. In Grierson, A.J.C. \& D.G. Long (eds.). Flora of Bhutan Including a Record of Plants from Sikkim. Royal Botanic Garden, Edinburgh, 272 pp.

Grierson, A.J.C. (1987). Family Crassulaceae, pp. 471-484. In: Grierson, A.J.C. \& D.G. Long (eds.). Flora of Bhutan Including a Record of Plants from Sikkim volume 1 (Part 3). Royal Botanic Garden, Edinburgh, $366 \mathrm{pp}$.

Grierson, A.J.C. (1987). Family Saxifragaceae, pp. 485-514. In: Grierson, A.J.C. \& D.G. Long (eds.). Flora of Bhutan Including a Record of Plants from Sikkim volume 1 (Part 3). Royal Botanic Garden, Edinburgh, 366 pp.

Hajra, P.K. \& D.M. Verma (Eds.) (1996). Flora of Sikkim (Vol. 1).
Botanical Survey of India, Calcutta, 307 pp.

Hooker, J.D. (1872-1897). The Flora of British India - Vol. 1-7. L. Reeve $\&$ Co. Ltd. The Oast House. Brook, Nr. Ashford,Kent.

Lahiri, S. \& S.S. Dash (2020). Lectotypification of Three Names in the Genus Gentiana (Gentianaceae) of Sikkim, India. Journal of Japanese Botany 95(2): 106-110.

Lahiri, S., S.S. Dash, A. Ghosh \& B.K. Sinha (2019). A contribution to the flora of Kanchenjunga Biosphere Reserve, Sikkim, India. Nelumbo 61(1): 17-26.

Long D.G. (1984). Family Papaveraceae, pp. 400-410. In: Grierson, A.J.C. \& D.G. Long (eds.). Flora of Bhutan Including a Record of Plants from Sikkim Volume 1 (Part 2). Royal Botanic Garden, Edinburgh, $272 \mathrm{pp}$.

Lucksom, S.Z. (2007). The Orchids of Sikkim and North East Himalaya. Siliguri, $984 \mathrm{pp}$.

Maity, D., G.G. Maiti \& A.S. Chauhan (2018). Vascular plants of Kanchenjunga Biosphere Reserve, Sikkim. Botanical Survey of India. Kolkata, India, 953 pp.

Mao, A.A., S.S. Dash \& P. Singh (2017). Rhododendrons of northeast India: A Pictorial Handbook. Botanical Survey of India, CGO complex, Kolkata, India, $167 \mathrm{pp}$.

Powo (2019). "Plants of the World Online. Facilitated by the Royal Botanic Gardens, Kew. Published on the Internet; http://www. plantsoftheworldonline.org. Accessed on 22 March 2020.

Singh, P. \& S.S. Dash (2015). Plant Discoveries 2014 - New Genera, Species and New Records. Botanical Survey of India, CGO complex, Kolkata, India, $142 \mathrm{pp}$.

Srivastava, R. C. (1998). Flora of Sikkim: Ranunculaceae-moringaceae. Oriental Enterprises.

WFO (2020). World Flora Online. http://www.worldfloraonline.org. Accessed on 20 March 2020 

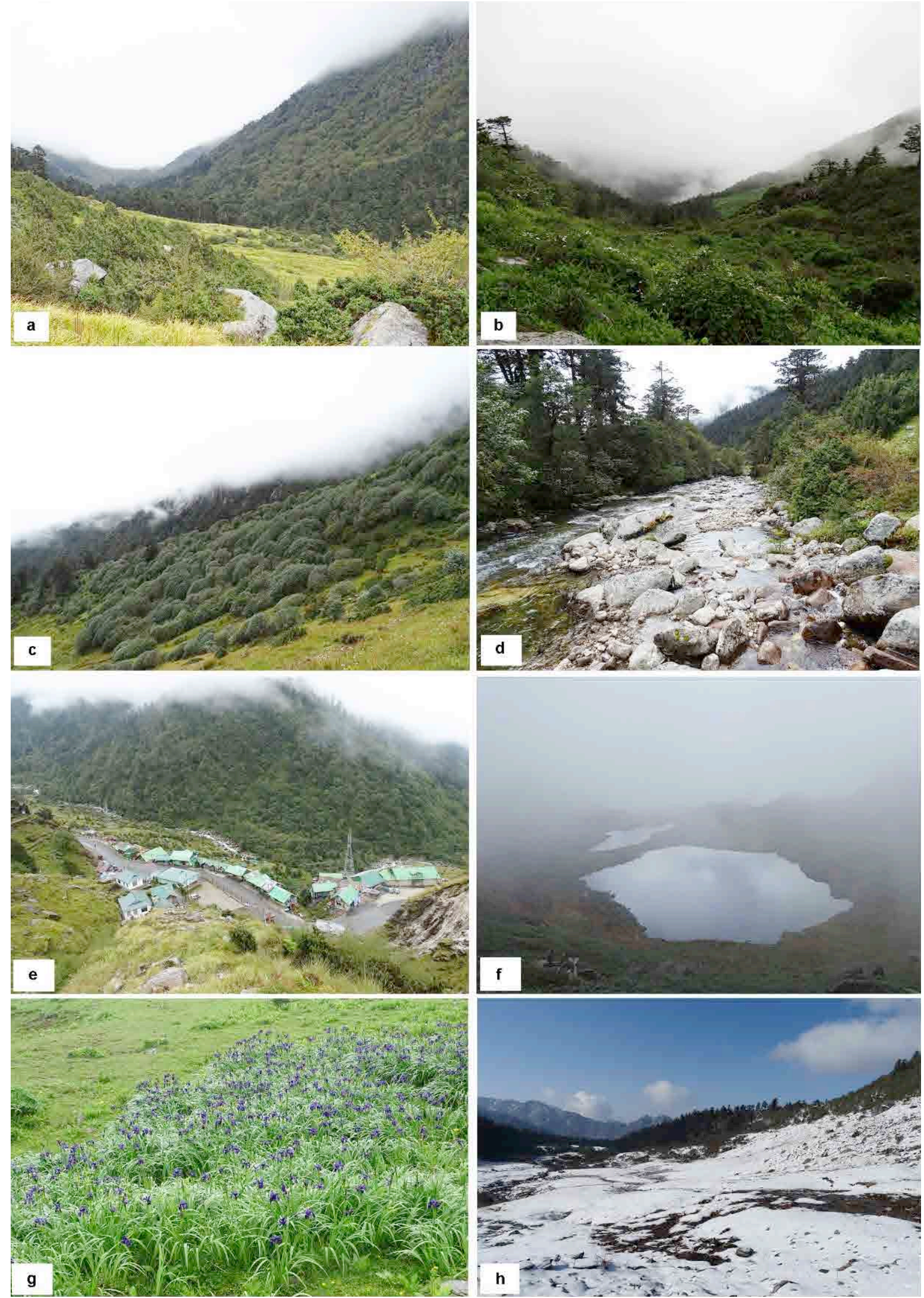

Image 1. a-Post monsoon view of Ek gote area at 3,300 $\mathrm{m} \mathrm{|} \mathrm{b-Rhododendron-Abies} \mathrm{forest} \mathrm{at} \mathrm{3,600} \mathrm{m} \mathrm{|} \mathrm{c-Rhododendron} \mathrm{woodland} \mathrm{at} \mathrm{Panch} \mathrm{gote}$ area at 3,800 m | d-Riverine habitat near Ek gote area at 3,300 m | e-View of Kyangnosla village in the sanctuary | $\mathrm{f}-$ Jor Pokhari Lake at Nakchok at 4,100 m | g-Gregarious growth of Iris clarkei at 4,122 m | h-Snow covered slope at Namnang (3,800 m) during autumn. @) Subhajit Lahiri. 

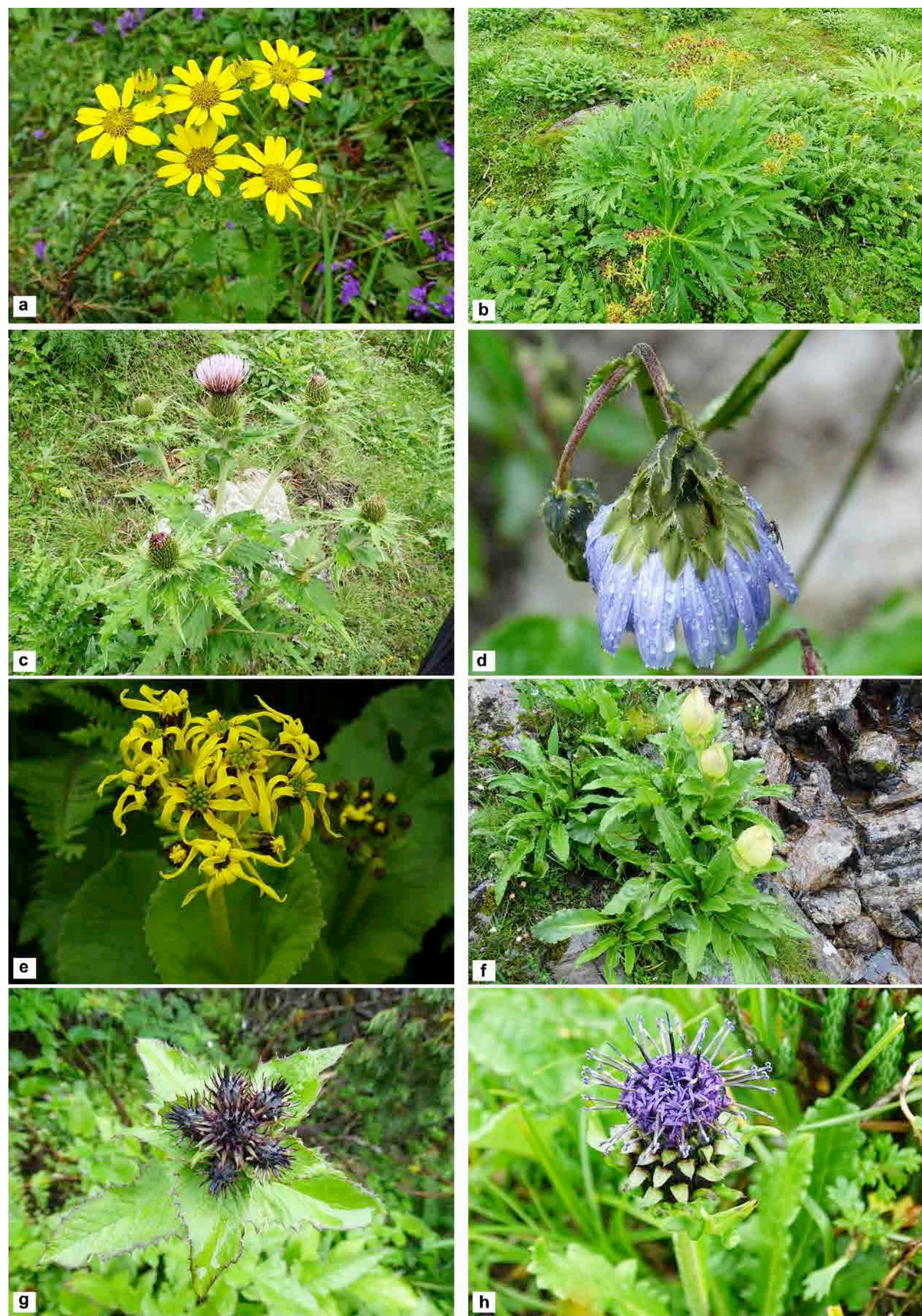

Image 2. a-Senecio raphanifolius Wall. ex DC. | b-Cacalia mortonii (C.B. Clarke) Kitam. ex H. Koyama | c-Cirsium falconeri (Hook.f.) Petrd. | d-Chaetoseris cyanea (D. Don) C. Shih | e-Senecio graciliflorus (Wall.) DC. | f-Saussurea obvallata (DC.) Edgew. | g-Saussurea piptathera Edgew | h-Saussurea nepalensis Spreng. (C) Subhajit Lahiri. 

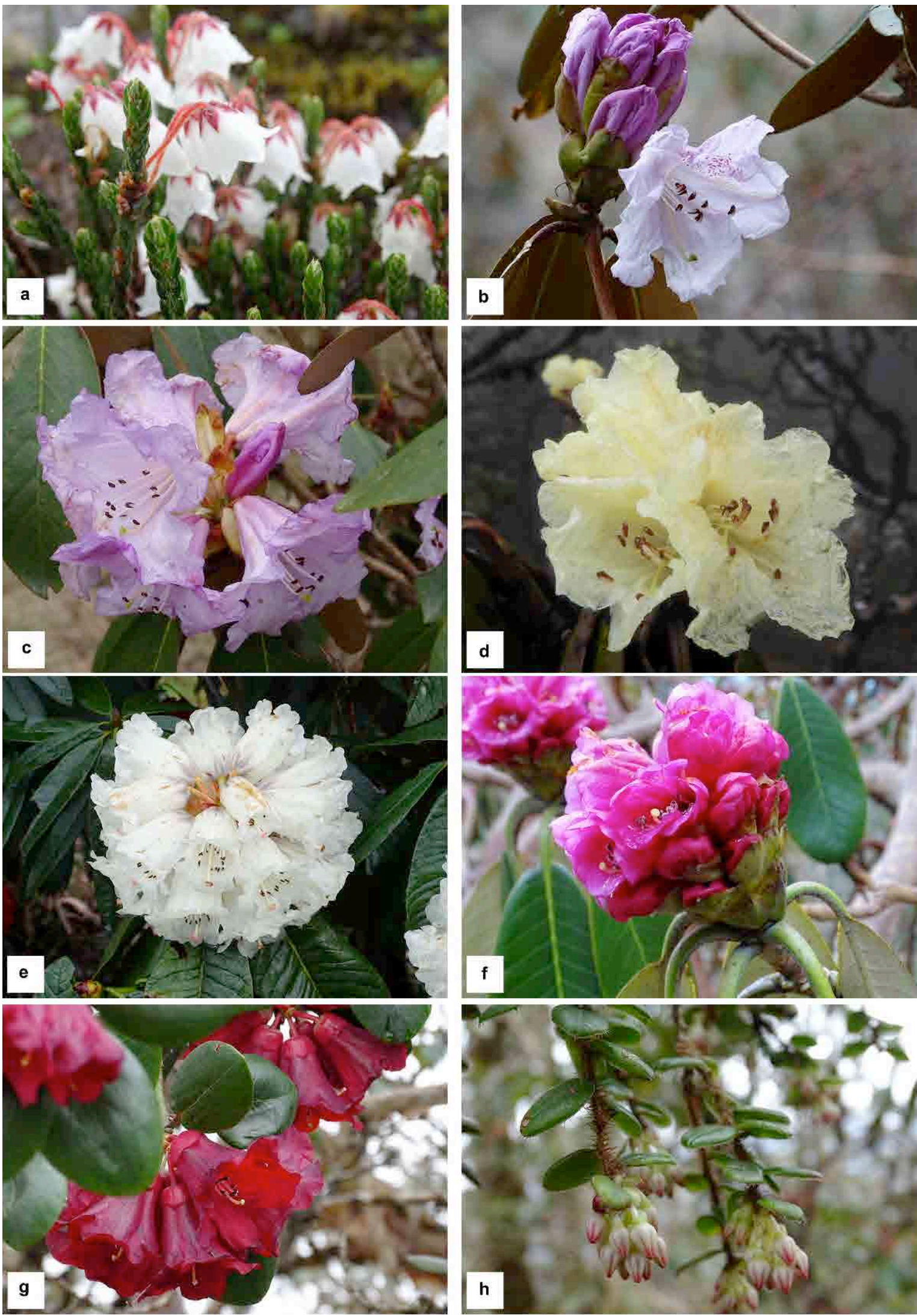

Image 3. a-Cassiope fastigiata (Wall.) D.Don | b-Rhododendron campanulatum D. Don | c-Rhododendron campanulatum subsp. aeruginosum (Hook.f.) D.F.Chamb. I d-Rhododendron campylocarpum Hook.f. | e-Rhododendron grande Wight | f-Rhododendron hodgsonii Hook.f. | g.-Rhododendron thomsonii Hook.f. | h-Vaccinium nummularia Hook.f. \& Thomson ex C.B. Clarke. @) Subhajit Lahiri. 

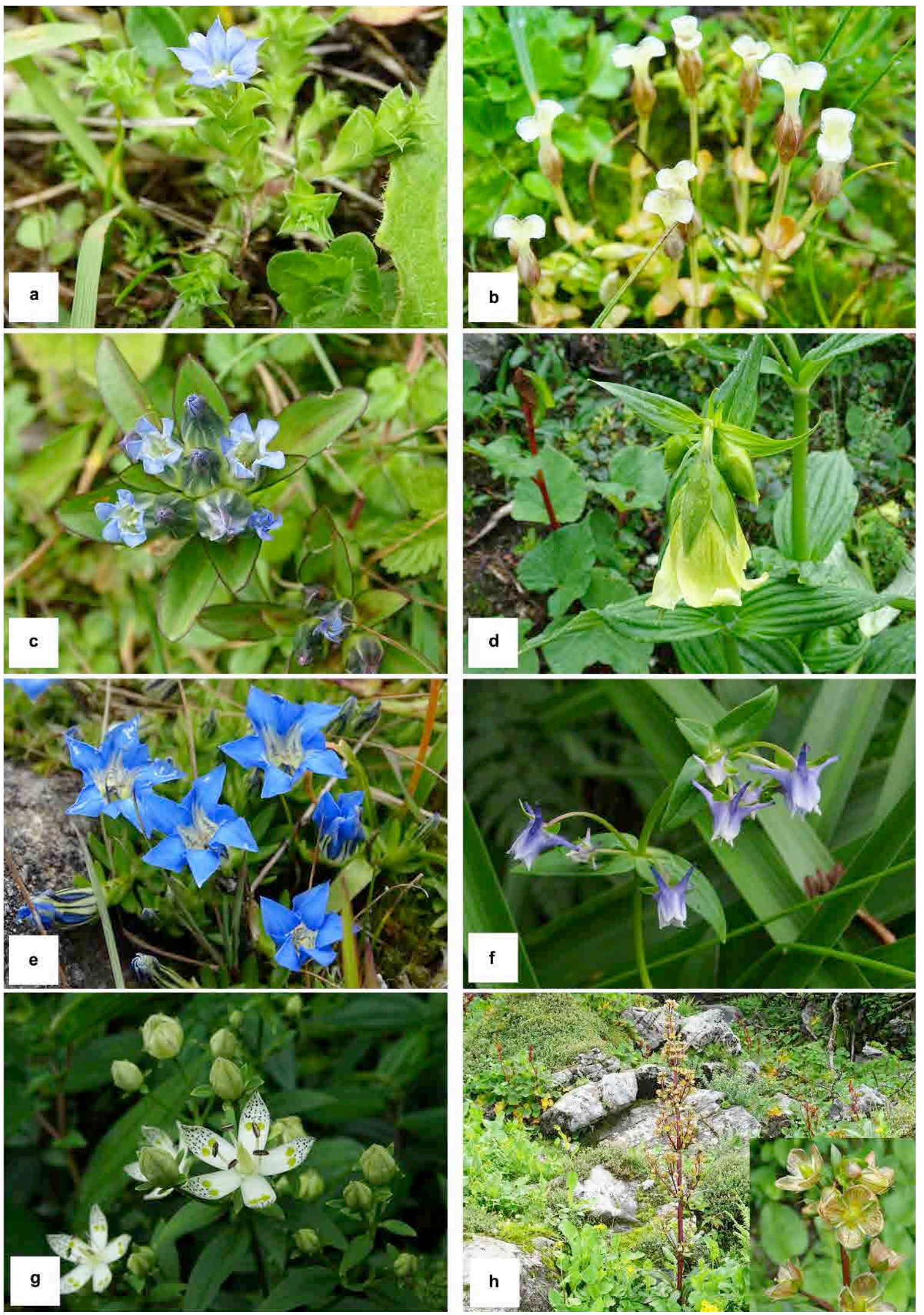

Image 4. a-Gentiana recurvata C.B.Clarke | b-Gentiana recurvata subsp. prainii (Burkill) Halda | c-Gentiana sikkimensis C.B.Clarke | dGentiana stylophora C.B.Clarke | e-Gentiana tubiflora (G.Don) Griseb | f-Halenia elliptica D.Don | g-Swertia bimaculata (Siebold \& Zucc.) Hook.f. \& Thomson ex C.B. Clarke | h-Swertia hookeri C.B. Clarke. @ Subhajit Lahiri. 

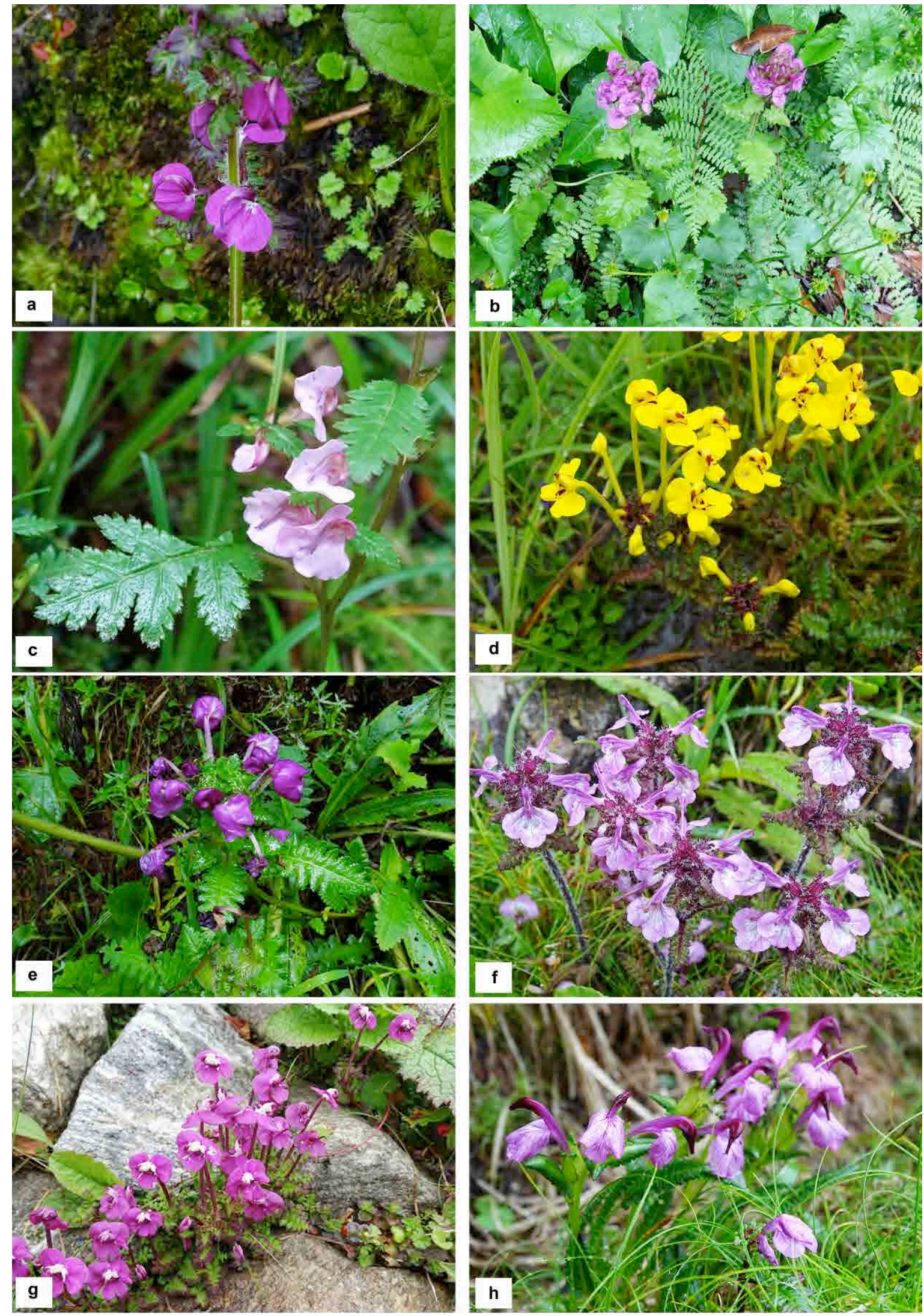

Image 5. a-Pedicularis flexuosa Hook.f | b-Pedicularis furfuracea Wall | c-Pedicularis gracilis subsp. macrocarpa (Prain) Tsoong | dPedicularis longiflora var. tubiformis (Klotzsch) Tsoong | e-Pedicularis megalantha D.Don | f-Pedicularis roylei Maxim | g-Pedicularis siphonantha D.Don | h-Pedicularis wallichii Bunge. @ Subhajit Lahiri. 

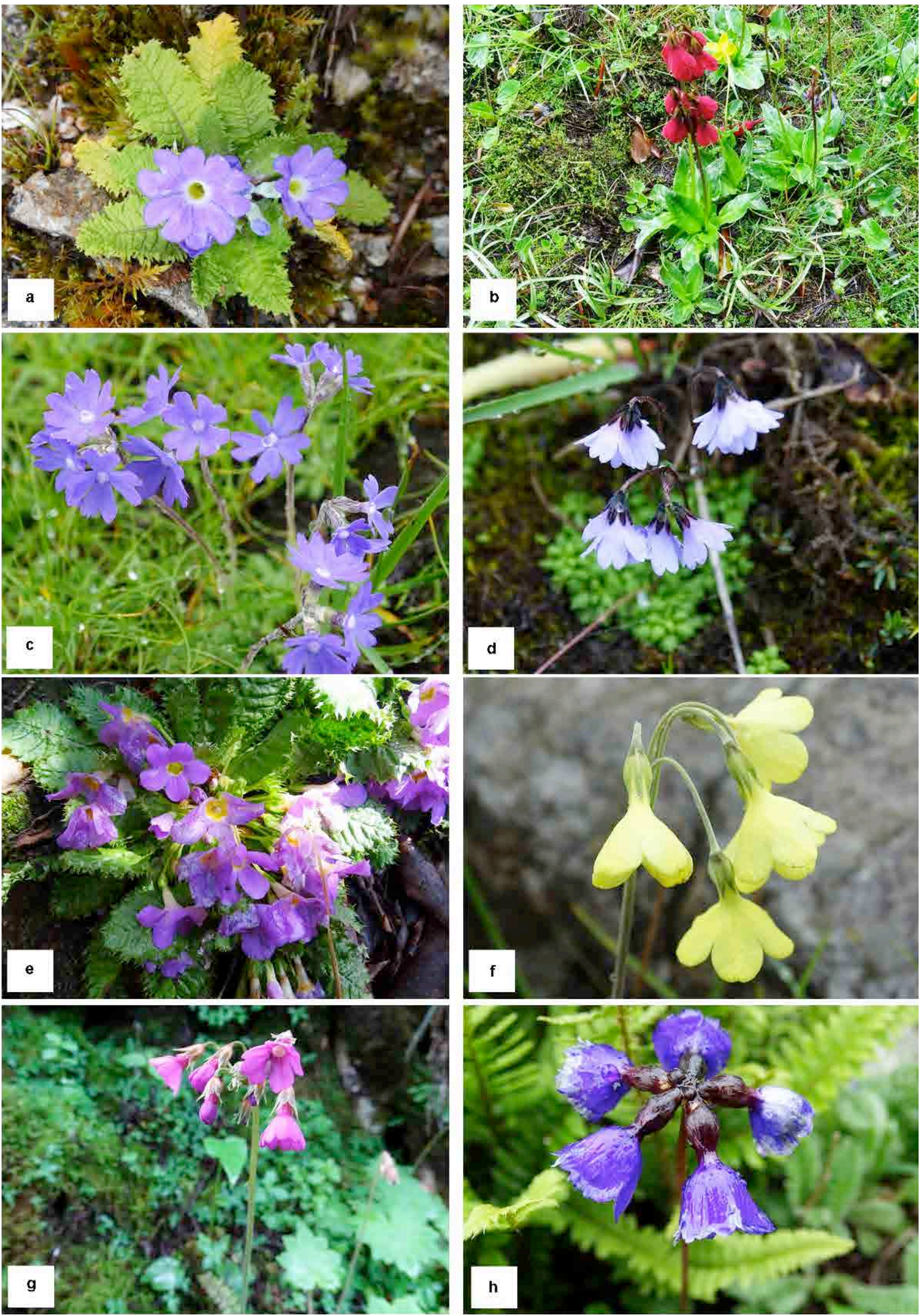

Image 6. a-Primula drummondiana Craib | b-Primula kingii Watt | c-Primula primulina (Spreng.) H.Hara | d-Primula sapphirina Hook.f. \& Thomson | e-Primula scapigera (Hook.f.) Craib | f-Primula sikkimensis Hook.f | g-Primula vaginata Watt | h-Primula wattii King ex Watt. @ Subhajit Lahiri. 
Dr. John Noyes, Natural History Museum, London, UK

Dr. Albert G. Orr, Griffith University, Nathan, Australia

Dr. Sameer Padhye, Katholieke Universiteit Leuven, Belgium

Dr. Nancy van der Poorten, Toronto, Canada

Dr. Kareen Schnabel, NIWA, Wellington, New Zealand

Dr. R.M. Sharma, (Retd.) Scientist, Zoological Survey of India, Pune, India

Dr. Manju Siliwal, WILD, Coimbatore, Tamil Nadu, India

Dr. G.P. Sinha, Botanical Survey of India, Allahabad, India

Dr. K.A. Subramanian, Zoological Survey of India, New Alipore, Kolkata, India

Dr. P.M. Sureshan, Zoological Survey of India, Kozhikode, Kerala, India

Dr. R. Varatharajan, Manipur University, Imphal, Manipur, India

Dr. Eduard Vives, Museu de Ciències Naturals de Barcelona, Terrassa, Spain

Dr. James Young, Hong Kong Lepidopterists' Society, Hong Kong

Dr. R. Sundararaj, Institute of Wood Science \& Technology, Bengaluru, India

Dr. M. Nithyanandan, Environmental Department, La Ala Al Kuwait Real Estate. Co. K.S.C.,

Kuwait

Dr. Himender Bharti, Punjabi University, Punjab, India

Mr. Purnendu Roy, London, UK

Dr. Saito Motoki, The Butterfly Society of Japan, Tokyo, Japan

Dr. Sanjay Sondhi, TITLI TRUST, Kalpavriksh, Dehradun, India

Dr. Nguyen Thi Phuong Lien, Vietnam Academy of Science and Technology, Hanoi, Vietnam

Dr. Nitin Kulkarni, Tropical Research Institute, Jabalpur, India

Dr. Robin Wen Jiang Ngiam, National Parks Board, Singapore

Dr. Lional Monod, Natural History Museum of Geneva, Genève, Switzerland.

Dr. Asheesh Shivam, Nehru Gram Bharti University, Allahabad, India

Dr. Rosana Moreira da Rocha, Universidade Federal do Paraná, Curitiba, Brasi

Dr. Kurt R. Arnold, North Dakota State University, Saxony, Germany

Dr. James M. Carpenter, American Museum of Natural History, New York, USA

Dr. David M. Claborn, Missouri State University, Springfield, USA

Dr. Kareen Schnabel, Marine Biologist, Wellington, New Zealand

Dr. Amazonas Chagas Júnior, Universidade Federal de Mato Grosso, Cuiabá, Brasil

Mr. Monsoon Jyoti Gogoi, Assam University, Silchar, Assam, India

Dr. Heo Chong Chin, Universiti Teknologi MARA (UiTM), Selangor, Malaysia

Dr. R.J. Shiel, University of Adelaide, SA 5005, Australia

Dr. Siddharth Kulkarni, The George Washington University, Washington, USA

Dr. Priyadarsanan Dharma Rajan, ATREE, Bengaluru, India

Dr. Phil Alderslade, CSIRO Marine And Atmospheric Research, Hobart, Australia

Dr. John E.N. Veron, Coral Reef Research, Townsville, Australia

Dr. Daniel Whitmore, State Museum of Natural History Stuttgart, Rosenstein, Germany.

Dr. Yu-Feng Hsu, National Taiwan Normal University, Taipei City, Taiwan

Dr. Keith V. Wolfe, Antioch, California, USA

Dr. Siddharth Kulkarni, The Hormiga Lab, The George Washington University, Washington,

D.C., USA

Dr. Tomas Ditrich, Faculty of Education, University of South Bohemia in Ceske

Budejovice, Czech Republic

Dr. Mihaly Foldvari, Natural History Museum, University of Oslo, Norway

Dr. V.P. Uniyal, Wildlife Institute of India, Dehradun, Uttarakhand 248001, India

Dr. John T.D. Caleb, Zoological Survey of India, Kolkata, West Bengal, India

Dr. Priyadarsanan Dharma Rajan, Ashoka Trust for Research in Ecology and the Environment

(ATREE), Royal Enclave, Bangalore, Karnataka, India

\section{Fishes}

Dr. Neelesh Dahanukar, IISER, Pune, Maharashtra, India

Dr. Topiltzin Contreras MacBeath, Universidad Autónoma del estado de Morelos, México

Dr. Heok Hee Ng, National University of Singapore, Science Drive, Singapore

Dr. Rajeev Raghavan, St. Albert's College, Kochi, Kerala, India

Dr. Robert D. Sluka, Chiltern Gateway Project, A Rocha UK, Southall, Middlesex, UK

Dr. E. Vivekanandan, Central Marine Fisheries Research Institute, Chennai, India

Dr. Davor Zanella, University of Zagreb, Zagreb, Croatia

Dr. A. Biju Kumar, University of Kerala, Thiruvananthapuram, Kerala, India

Dr. Akhilesh K.V., ICAR-Central Marine Fisheries Research Institute, Mumbai Research

Centre, Mumbai, Maharashtra, India

Dr. J.A. Johnson, Wildlife Institute of India, Dehradun, Uttarakhand, India

Amphibians

Dr. Sushil K. Dutta, Indian Institute of Science, Bengaluru, Karnataka, India

Dr. Annemarie Ohler, Muséum national d'Histoire naturelle, Paris, France

\section{Reptiles}

Dr. Gernot Vogel, Heidelberg, Germany

Dr. Raju Vyas, Vadodara, Gujarat, India

Dr. Pritpal S. Soorae, Environment Agency, Abu Dubai, UAE.

Prof. Dr. Wayne J. Fuller, Near East University, Mersin, Turkey

Prof. Chandrashekher U. Rivonker, Goa University, Taleigao Plateau, Goa. India

Dr. S.R. Ganesh, Chennai Snake Park, Chennai, Tamil Nadu, India

Dr. Himansu Sekhar Das, Terrestrial \& Marine Biodiversity, Abu Dhabi, UAE
Birds

Dr. Hem Sagar Baral, Charles Sturt University, NSW Australia

Dr. Chris Bowden, Royal Society for the Protection of Birds, Sandy, UK

Dr. Priya Davidar, Pondicherry University, Kalapet, Puducherry, India

Dr. J.W. Duckworth, IUCN SSC, Bath, UK

Dr. Rajah Jayapal, SACON, Coimbatore, Tamil Nadu, India

Dr. Rajiv S. Kalsi, M.L.N. College, Yamuna Nagar, Haryana, India

Dr. V. Santharam, Rishi Valley Education Centre, Chittoor Dt., Andhra Pradesh, India

Dr. S. Balachandran, Bombay Natural History Society, Mumbai, India

Mr. J. Praveen, Bengaluru, India

Dr. C. Srinivasulu, Osmania University, Hyderabad, India

Dr. K.S. Gopi Sundar, International Crane Foundation, Baraboo, USA

Dr. Gombobaatar Sundev, Professor of Ornithology, Ulaanbaatar, Mongolia

Prof. Reuven Yosef, International Birding \& Research Centre, Eilat, Israel

Dr. Taej Mundkur, Wetlands International, Wageningen, The Netherlands

Dr. Carol Inskipp, Bishop Auckland Co., Durham, UK

Dr. Tim Inskipp, Bishop Auckland Co, Durham, UK

Dr. V. Gokula, National College, Tiruchirappalli, Tamil Nadu, India

Dr. Arkady Lelej, Russian Academy of Sciences, Vladivostok, Russia

Dr. Simon Dowell, Science Director, Chester Zoo, UK

Dr. Mário Gabriel Santiago dos Santos, Universidade de Trás-os-Montes e Alto Douro,

Quinta de Prados, Vila Real, Portugal

Dr. Grant Connette, Smithsonian Institution, Royal, VA, USA

Dr. M. Zafar-ul Islam, Prince Saud Al Faisal Wildlife Research Center, Taif, Saudi Arabia

Mammals

Dr. Giovanni Amori, CNR - Institute of Ecosystem Studies, Rome, Italy

Dr. Anwaruddin Chowdhury, Guwahati, India

Dr. David Mallon, Zoological Society of London, UK

Dr. Shomita Mukherjee, SACON, Coimbatore, Tamil Nadu, India

Dr. Angie Appel, Wild Cat Network, Germany

Dr. P.O. Nameer, Kerala Agricultural University, Thrissur, Kerala, India

Dr. Ian Redmond, UNEP Convention on Migratory Species, Lansdown, UK

Dr. Heidi S. Riddle, Riddle's Elephant and Wildlife Sanctuary, Arkansas, USA

Dr. Karin Schwartz, George Mason University, Fairfax, Virginia.

Dr. Lala A.K. Singh, Bhubaneswar, Orissa, India

Dr. Mewa Singh, Mysore University, Mysore, India

Dr. Paul Racey, University of Exeter, Devon, UK

Dr. Honnavalli N. Kumara, SACON, Anaikatty P.O., Coimbatore, Tamil Nadu, India

Dr. Nishith Dharaiya, HNG University, Patan, Gujarat, India

Dr. Spartaco Gippoliti, Socio Onorario Società Italiana per la Storia della Fauna "Giuseppe

Altobello", Rome, Italy

Dr. Justus Joshua, Green Future Foundation, Tiruchirapalli, Tamil Nadu, India

Dr. H. Raghuram, The American College, Madurai, Tamil Nadu, India

Dr. Paul Bates, Harison Institute, Kent, UK

Dr. Jim Sanderson, Small Wild Cat Conservation Foundation, Hartford, USA

Dr. Dan Challender, University of Kent, Canterbury, UK

Dr. David Mallon, Manchester Metropolitan University, Derbyshire, UK

Dr. Brian L. Cypher, California State University-Stanislaus, Bakersfield, CA

Dr. S.S. Talmale, Zoological Survey of India, Pune, Maharashtra, India

Prof. Karan Bahadur Shah, Budhanilakantha Municipality, Kathmandu, Nepal

Dr. Susan Cheyne, Borneo Nature Foundation International, Palangkaraja, Indonesia

Dr. Hemanta Kafley, Wildlife Sciences, Tarleton State University, Texas, USA

\section{Other Disciplines}

Dr. Aniruddha Belsare, Columbia MO 65203, USA (Veterinary)

Dr. Mandar S. Paingankar, University of Pune, Pune, Maharashtra, India (Molecular)

Dr. Jack Tordoff, Critical Ecosystem Partnership Fund, Arlington, USA (Communities)

Dr. Ulrike Streicher, University of Oregon, Eugene, USA (Veterinary)

Dr. Hari Balasubramanian, EcoAdvisors, Nova Scotia, Canada (Communities)

Dr. Rayanna Hellem Santos Bezerra, Universidade Federal de Sergipe, São Cristóvão, Brazil

Dr. Jamie R. Wood, Landcare Research, Canterbury, New Zealand

Dr. Wendy Collinson-Jonker, Endangered Wildlife Trust, Gauteng, South Africa

Dr. Rajeshkumar G. Jani, Anand Agricultural University, Anand, Gujarat, India

Dr. O.N. Tiwari, Senior Scientist, ICAR-Indian Agricultural Research Institute (IARI), New

Delhi, India

Dr. L.D. Singla, Guru Angad Dev Veterinary and Animal Sciences University, Ludhiana, India

Dr. Rupika S. Rajakaruna, University of Peradeniya, Peradeniya, Sri Lanka

Dr. Bahar Baviskar, Wild-CER, Nagpur, Maharashtra 440013, India

Reviewers 2018-2020

Due to pausity of space, the list of reviewers for $2018-2020$ is available online.

The opinions expressed by the authors do not reflect the views of the Journal of Threatened Taxa, Wildlife Information Liaison Development Society, Zoo Outreach Organization, or any of the partners. The journal, the publisher, the host, and the partners are not responsible for the accuracy of the political boundaries shown in the maps by the authors.

Journal of Threatened Taxa is indexed/abstracted in Bibliography of Systematic Mycology, Biological Abstracts, BIOSIS Previews, CAB Abstracts, EBSCO, Google Scholar, Index Copernicus, Index Fungorum, JournalSeek, National Academy of Agricultural Sciences, NewJour, OCLC WorldCat, SCOPUS, Stanford University Libraries, Virtual Library of Biology, Zoological Records.

NAAS rating (India) 5.64
Print copies of the Journal are available at cost. Write to:

The Managing Editor, JoTT,

c/o Wildlife Information Liaison Development Society,

No. 12, Thiruvannamalai Nagar, Saravanampatti - Kalapatti Road,

Saravanampatti, Coimbatore, Tamil Nadu 641035, India

ravi@threatenedtaxa.org 


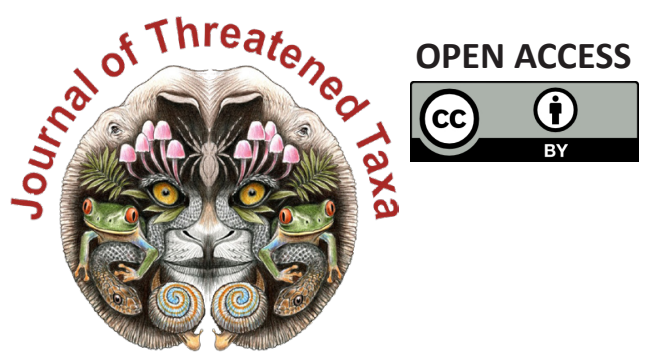

www.threatenedtaxa.org

The Journal of Threatened Taxa (JoTT) is dedicated to building evidence for conservation globally by publishing peer-reviewed articles online every month at a reasonably rapid rate at www.threatenedtaxa.org. All articles published in JoTT are registered under Creative Commons Attribution 4.0 International License unless otherwise mentioned. JoTT allows allows unrestricted use, reproduction, and distribution of articles in any medium by providing adequate credit to the author(s) and the source of publication.

ISSN 0974-7907 (Online) I ISSN $0974-7893$ (Print)

\section{November 2021 | Vol. 13 | No. 13 | Pages: 19887-20142 \\ Date of Publication: 26 November 2021 (Online \& Print) DOI: 10.11609/jott.2021.13.13.19887-20142}

\section{Article}

An inventory of geometrid moths (Lepidoptera: Geometroidea: Geometridae) of KalakadMundanthurai Tiger Reserve, India

- Geetha Iyer, Dieter Stüning \& Sanjay Sondhi, Pp. 19887-19920

\section{Communications}

Roadkills of Lowland Tapir Tapirus terrestris (Mammalia: Perissodactyla: Tapiridae) in one of its last refuges in the Atlantic Forest

- Aureo Banhos, Andressa Gatti, Marcelo Renan de Deus Santos, Leonardo Merçon,

Ilka Westermeyer, Natália Carneiro Ardente, Luis Francisco Oliveira Pereira Gonzaga, Lucas Mendes Barreto, Lucas Damásio, Tomas Lima Rocha, Vitor Roberto Schettino, Renata Valls, Helena Godoy Bergallo, Marcos Vinicius Freitas Silva, Athelson Stefanon Bittencourt, Danielle de Oliveira Moreira \& Ana Carolina Srbek-Araujo, Pp. 19921-19929

Scientific contributions and learning experiences of citizen volunteers with a small cat project in Sanjay Gandhi National Park, Mumbai, India

- Shomita Mukherjee, R. Nandini, P.V. Karunakaran \& Nayan Khanolkar, Pp. 19930-19936

Seasonal food preferences and group activity pattern of Blackbuck Antilope cervicapra (L., 1758) (Mammalia: Cetartiodactyla: Bovidae) in a semi-arid region of western Haryana, India

- Vikram Delu, Dharambir Singh, Sumit Dookia, Priya \& Kiran, Pp. 19937-19947

Studies on the habitats of Grey Francolin Francolinus pondicerianus (J.F. Gmelin, 1789) (Galliformes: Phasianidae) in northern districts of Tamil Nadu, India

- M. Pandian, Pp. 19948-19955

Recovery of vulture population in roosting and scavenging areas of Bastar and Bijapur, Chhattisgarh, India

- Sushil Kumar Dutta, Muntaz Khan, P.R.S. Nagi, Santosh Durgam \& Surabhi Dutta, Pp. 19956-19963

A geographical assessment of Chariganga and Arpara Beel (wetlands) of Nadia, West Bengal as a habitat of wetland birds

- Mehedi Hasan Mandal, Arindam Roy \& Giyasuddin Siddique, Pp. 19964-19975

Phenotypic plasticity in Barilius vagra (Hamilton, 1822) (Teleostei: Danionidae) from two geographically distinct river basins of Indian Himalaya

- Sumit Kumar, Sharali Sharma \& Deepak Singh, Pp. 19976-19984

Taxonomic notes, a new species, and a key to Indian species of the click beetle genus Cryptalaus Ôhira, 1967 (Coleoptera: Elateridae: Agrypninae)

- Harshad Parekar \& Amol Patwardhan, Pp. 19985-19999

Niche overlap of benthic macrofauna in a tropical estuary: diurnal variation

- Mário Herculano de Oliveira, Lidiane Gomes de Lima, Caroline Stefani da Silva Lima, Jéssica de Oliveira Lima Gomes, Franciely Ferreira Paiva, Graciele de Barros, Carlinda Railly Medeiros \& Joseline Molozzi, Pp. 20000-20010

Diversity of aquatic insects and biomonitoring of water quality in the upper Ganga River, a Ramsar site: a preliminary assessment

- Kritish De, Arkojyoti Sarkar, Kritika Singh, Virendra Prasad Uniyal, Jeyaraj Antony Johnson \& Syed Ainul Hussain, Pp. 20011-20018

Patterns of forest cover loss in the terrestrial Key Biodiversity Areas in the Philippines: critical habitat conservation priorities

- Bernard Peter O. Daipan, Pp. 20019-20032

The woody flora of Shettihalli Wildlife Sanctuary, central Western Ghats of Karnataka, India - A checklist

- Kanda Naveen Babu, Kurian Ayushi, Vincy K. Wilson, Narayanan Ayyappan \&

Narayanaswamy Parthasarathy, Pp. 20033-20055

Reproductive biology of Ophiorrhiza caudata C.E.C.Fisch. (Rubiaceae), an endemic and endangered creeping perennial herb of the Western Ghats, India

- Maria Theresa, Appukuttan Kamalabai Sreekala \& Jayalakshmi Mohanlal, Pp. 20056-20065
Short Communications

Successful rescue, medical management, rehabilitation, and translocation of a Red Panda Ailurus fulgens (Mammalia: Carnivora: Ailuridae) in Arunachal Pradesh, India - Jahan Ahmed, Sorang Tadap, Millo Tasser, Koj Rinya, Nekibuddin Ahmed \& Sunil Kyarong, Pp. 20066-20071

A rare photographic record of Eurasian Otter Lutra lutra with a note on its habitat from the Bhagirathi Basin, western Himalaya, India

- Ranjana Pal, Aashna Sharma, Vineet Kumar Dubey, Tapajit Bhattacharya, Jeyaraj Antony Johnson, Kuppusamy Sivakumar \& Sambandam Sathyakumar, Pp. 20072-20077

The first record of Medog Gliding Frog Rhacophorus translineatus Wu, 1977 (Anura: Rhacophoridae) from Chhukha District, Bhutan

- Sonam Lhendup \& Bal Krishna Koirala, Pp. 20078-20083

First record of a freshwater crab, Maydelliathelphusa masoniana (Henderson, 1893) (Decapoda: Brachyura: Gecarcinucidae) from West Bengal, India

- Ram Krishna Das, Pp. 20084-20089

Butterflies of Amrabad Tiger Reserve, Telangana, India

- Deepa Jaiswal, B. Bharath, M. Karuthapandi, Shrikant Jadhav, S. Prabakaran \& S. Rehanuma Sulthana, Pp. 20090-20097

An enumeration of the flowering plants of Kyongnosla Alpine Sanctuary in eastern Sikkim, India

- Sudhansu Sekhar Dash, Subhajit Lahiri \& Ashiho Asoshii Mao, Pp. 20098-20117

A new record of psychrotrophic Paecilomyces formosus (Eurotiales: Ascomycota) from India: morphological and molecular characterization

- Skarma Nonzom \& Geeta Sumbali, Pp. 20118-20123

Notes

Study on incidence and pathology of gastrointestinal parasitic infections in Nilgai Boselaphus tragocamelus in Hisar, Haryana, India

- Maneesh Sharma, B.L. Jangir, D. Lather, G.A. Chandratre, V. Nehra, K.K. Jakhar \& G. Narang, Pp. 20124-20127

An unusual vocalization of Brown Hawk-Owl Ninox scutulata (Raffles, 1822) (Aves:

Strigiformes: Strigidae) recorded from Kerala, India

- Riju P. Nair \& Shine Raj Tholkudiyil, Pp. 20128-20129

New distribution data on the genus Maripanthus Maddison, 2020 (Araneae: Salticidae) from southern India

- A. Asima, John T.D. Caleb, Dhruv A. Prajapati \& G. Prasad, Pp. 20130-20132

On the IUCN status of Boesenbergia albolutea and B. rubrolutea (Zingiberaceae) and typification of $B$. rubrolutea

- K. Aishwarya \& M. Sabu, Pp. 20133-20135

New records of mass seeding Cephalostachyum latifolium Munro (Poaceae) along the midelevation broadleaved forest of Sarpang district, Bhutan

- Jigme Tenzin, Sangay Nidup \& Dago Dorji, Pp. 20136-20139

Response

If habitat heterogeneity is effective for conservation of butterflies in urban landscapes of Delhi, India?' Unethical publication based on data manipulation

- Sanjay Keshari Das \& Rita Singh, Pp. 20140-20142

Publisher \& Host
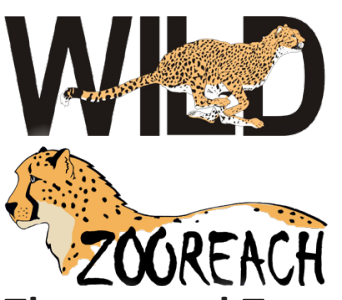

Threatened Taxa 